\title{
14. COMMISSION DES ETALONS DE LONGUEUR D'ONDE ET DES TABLES DE SPECTRES SOLAIRES
}

Président: M. W. F. Meggers, National Bureau of Standards, Washington, D.C. Membres: MM. Babcock, Buisson, Burns, Ch. Fabry, A. Fowler, Harrison, Kayser, Nagaoka, Pérard.

\section{THE PRIMARY STANDARD}

The red radiation, 6438.4696 A., emitted by a cadmium lamp of Michelson type was first chosen in 1907 by the International Union for Co-operation in Solar Research (Trans. I.U.S.R. 2, I09, I907) as a definition of the unit of wave-length. This primary standard was subsequently adopted by the International Astronomical Union (Trans. I.A.U. I, 35, I922) and by the International Committee on Weights and Measures (Procès-Verbaux Comité Int. Poids et Mesures (2), 12, 67, 1927). Specifications for the production of this primary standard were adopted provisionally by the I.A.U. in I925 (Trans. I.A.U. 2, 47, 232, I925), and by the I.C.W.M. in I927 (Proces-Verbaux Comité Int. Poids et Mesures (2), 12, 67, 1927). Three reports of this Commission (Trans. I.A.U. 3, 77, 236, 1928; ibid. 4, 58, 233, 1932; ibid. 5, 8I, 299, I935) have discussed the divergences in these specifications and pointed out the unsatisfactory features of each. This discussion culminated in a revised specification (Trans. I.A.U. 5, 303, 1935) which was adopted unanimously by the I.C.W.M. in 1935 (Proces-Verbaux Comité Int. (2), 17, 9I, 1935). Since Commission I4 in its last meeting decided to defer further action on this matter until the I.C.W.M. had made its recommendation, it now seems appropriate to recommend that the I.A.U. adopt the identical specification, which is as follows:

"Spécifications pour la lampe à cadmium du type Michelson. Pour émettre dans des conditions favorables le raie primaire des longueurs d'onde lumineuses $\lambda=6438 \cdot 4696$ UA, la lampe à cadmium du type Michelson, comportant des électrodes intérieures et excitée par courant électrique, continu, ou alternatif de fréquence industrielle, doit être maintenue à une température voisine de $300^{\circ} \mathrm{C}$. (en tout cas ne dépassant pas $320^{\circ}$ ) et contenir de l'air sous une pression comprise entre $0.7 \mathrm{~mm}$. et I mm. de mercure à cette température. Si elle présente un tube capillaire ou plus généralement un étranglement destiné à augmenter sa brillance, aucune dimension latérale de cet étranglement ne devra être inférieure à $2 \mathrm{~mm}$. L'intensité du courant d'excitation ne dépassera pas une valeur telle que sa densité risque d'atteindre $7 \mathrm{~mA}$ par millimètre carré de la section la plus étroite de la région observée."

\section{SECONDARY STANDARDS. IRON LINES}

Except for three iron lines adopted in 1932 (Trans. I.A.U. 4, 234, 1932) no additions or extensions have been made to the list of 235 secondary standards adopted in I928 (Trans. I.A.U. 3, 86, I928). These extend from 3370.787 to $6677.993 \mathrm{~A}$., thus covering less than one octave of spectrum. Repeated recommendations that this system be extended both to longer and to shorter waves have been responsible for interferometer measurements of iron lines in the infra-red and ultraviolet. Unfortunately, no efforts to confirm the infra-red values quoted in the last report (Trans. I.A.U. 5, 84, I935) have been announced and it is, therefore, impossible to extend the present system of secondary iron standards in this direction. 
However, a considerable extension of secondary standards in the ultra-violet is now obtained by averaging the measurements made by Burns and Walters ( $P u b l$. Allegheny Obs. 6, 159, I929; ibid. 8, 39, I93I), by C. V. Jackson (Proc. Roy. Soc. (London), A, 130, 395, I93I; ibid. A, 133, 553, I93I), and by Meggers and Humphreys (J. Research, Nat. Bur. Stand. 18, 543, I937). These are displayed in Tables I and 2. The data published by Burns and Walters consist of vacuum arc values, those reported in 1929 being based on neon standards, or calculated from terms thus established, while those appearing in I93I were interpolated between values previously determined for iron and copper lines. Jackson compared iron lines from the Pfund arc directly with the primary standard or with standard neon lines, and similar measurements were made by Meggers and Humphreys relative to cadmium, neon, and krypton standards.

\section{TABLE I}

Wave-Lengths of Iron Arc Secondary Standards in Air at $15^{\circ} \mathrm{C}$. and $760 \mathrm{~mm}$. Pressure, 3497 to $3370 \mathrm{~A}$.

\begin{tabular}{|c|c|c|c|c|c|c|}
\hline \multirow[b]{2}{*}{$\underset{\lambda}{\text { Recommended }}$} & \multicolumn{2}{|c|}{ I.A.U. 1928} & \multirow{2}{*}{$\begin{array}{c}\text { Burns- } \\
\text { Walters } \\
\text { calc. }\end{array}$} & \multirow[b]{2}{*}{ Jackson } & \multirow[b]{2}{*}{$\begin{array}{l}\text { Meggers- } \\
\text { Humphreys }\end{array}$} & \multirow[b]{2}{*}{$\begin{array}{c}\text { Term } \\
\text { combination }\end{array}$} \\
\hline & Standard & $\begin{array}{l}\text { Inter- } \\
\text { polated }\end{array}$ & & & & \\
\hline $\begin{array}{r}\mathbf{3 4 9 7 \cdot 8 4 3} \\
\mathbf{3 4 9 0 \cdot 5 7 5} \\
\mathbf{3 4 8 5 \cdot 3 4 2} \\
\mathbf{3 4 7 6 \cdot 7 0 4} \\
\mathbf{3 4 6 5 \cdot 8 6 3}\end{array}$ & $\begin{array}{l}.844 \\
\frac{-}{.705} \\
.863\end{array}$ & $\begin{array}{l}\cdot 844 \\
.342 \\
.706 \\
.863\end{array}$ & $\begin{array}{l}\cdot 842 \\
.574 \\
.341 \\
.703 \\
\cdot 862\end{array}$ & $\begin{array}{l}\cdot 843 \\
\cdot 575 \\
\cdot 342 \\
\cdot 703 \\
\cdot 863\end{array}$ & $\begin{array}{l}.842 \\
.575 \\
.342 \\
.704 \\
.862\end{array}$ & $\begin{array}{l}a^{5} \mathrm{D}_{1}-a^{5} \mathrm{P}_{8}^{\circ} \\
a^{5} \mathrm{D}_{8}-a^{5} \mathrm{P}_{8}^{\circ} \\
a^{5} \mathrm{P}_{2}-a^{5} \mathrm{P}_{1}^{\circ} \\
a^{5} \mathrm{D}_{0}-a^{5} \mathrm{P}_{1}^{\circ} \\
a^{5} \mathrm{D}_{1}-a^{6} \mathrm{P}_{1}^{\circ}\end{array}$ \\
\hline $\begin{array}{l}\mathbf{3 4 4 5 \cdot 1 5 1} \\
3443 \cdot 878 \\
\mathbf{3 4 2 7} \cdot 121 \\
\mathbf{3 4 1 3} \cdot 135 \\
\mathbf{3 4 0 7} \cdot 461\end{array}$ & $\begin{array}{l}\bar{z} \\
\bar{z}\end{array}$ & $\begin{array}{l}\cdot 152 \\
\cdot \overline{121} \\
\cdot 135 \\
\cdot 462\end{array}$ & $\begin{array}{l}\cdot 151 \\
\cdot 878 \\
\cdot 120 \\
\cdot 135 \\
\cdot 461\end{array}$ & $\begin{array}{l}\cdot 150 \\
\cdot 878 \\
\cdot 121 \\
=\end{array}$ & $\begin{array}{l}\cdot 151 \\
\cdot 877 \\
\cdot 121 \\
\cdot 134 \\
\cdot 461\end{array}$ & $\begin{array}{l}a^{5} \mathrm{P}_{2}-19 \mathrm{R}_{8}{ }^{\circ} \\
a^{5} \mathrm{D}_{2}-a^{6} \mathrm{P}_{1}^{\circ} \\
a^{5} \mathrm{P}_{3}-18 \mathrm{R}_{4}^{\circ} \\
a^{5} \mathrm{P}_{2}-d^{3} \mathrm{D}_{8}^{\circ} \\
a^{5} \mathrm{P}_{3}-c^{3} \mathrm{~F}_{4}^{\circ}\end{array}$ \\
\hline $\begin{array}{l}\mathbf{3 4 0 1 \cdot 5 2 1} \\
\mathbf{3 3 9 9 \cdot 3 3 6} \\
\mathbf{3 3 9 6} \cdot 978 \\
\mathbf{3 3 8 3} \\
\mathbf{3 3 8 0}\end{array}$ & $\begin{array}{l}522 \\
- \\
-\end{array}$ & $\begin{array}{l}.522 \\
.336 \\
.980 \\
- \\
-\end{array}$ & $\begin{array}{r}.520 \\
.335 \\
.978 \\
.980 \\
(\cdot 110)\end{array}$ & $\begin{array}{l}.522 \\
.337 \\
- \\
-\end{array}$ & $\begin{array}{l}.520 \\
.334 \\
.977 \\
.981 \\
.111\end{array}$ & $\begin{array}{c}a^{5} \mathrm{~F}_{4}-b{ }^{5} \mathrm{P}_{8}{ }^{\circ} \\
a^{5} \mathrm{P}_{2}-d{ }^{3} \mathrm{D}_{8}^{\circ} \\
a^{6} \mathrm{~F}_{8}-b{ }^{5} \mathrm{P}_{2}{ }^{\circ} \\
a^{5} \mathrm{P}_{8}-c^{8} \mathrm{~F}_{8}^{\circ} \\
\text { - }\end{array}$ \\
\hline $3370 \cdot 786$ & .787 & $\cdot 787$ & $(\cdot 783)$ & $\cdot 786$ & .784 & - \\
\hline
\end{tabular}

It was shown in the I932 report (Trans. I.A.U. 4, 64, I932) that the spectralterm depressions measured by Babcock for a pressure of one atmosphere permitted the calculation of wave-lengths for the iron arc in air from those observed in the vacuum arc. The Burns and Walters values quoted in Tables $x$ and 2 have been calculated in this manner by applying the appropriate corrections to their vacuumarc terms, recalculating wave-numbers from these corrected terms and finally reading the wave-lengths in standard air from Kayser's Schwingungszahlen $(\mathrm{H}$. Kayser, Tabelle der Schwingungszahlen, S. Hirzel, Leipzig, I925; Phys. Rev. 48, 98, I935). Values in parentheses are quoted from vacuum-arc observations since data for their conversion to atmospheric-arc values are lacking either because the Fe I lines are unclassified or because they are Fe II lines for which no term depressions have been measured. When the proper corrections for these lines are determined it will be possible to derive several dozen additional ultra-violet standards from the means of three independent determinations. 


\section{TABLE 2}

Wave-Lengths of Iron Arc Secondary Standards in Air at $15^{\circ} \mathrm{C}$. and $760 \mathrm{~mm}$. Pressure, 3355 to 2 roo A.

\begin{tabular}{|c|c|c|c|c|}
\hline$\underset{\lambda}{\operatorname{Recommended}}$ & $\begin{array}{c}\text { Burns- } \\
\text { Walters } \\
\text { calc. }\end{array}$ & Jackson & $\begin{array}{l}\text { Meggers- } \\
\text { Humphreys }\end{array}$ & $\begin{array}{c}\text { Term } \\
\text { combination }\end{array}$ \\
\hline $\begin{array}{l}\mathbf{3 3 5 5} \\
\mathbf{3 3 4 7 \cdot 9 2 7} \\
\mathbf{3 3 4 0 \cdot 5 6 6} \\
\mathbf{3 3 3 7} \\
\mathbf{3 3 2 8}\end{array}$ & $\begin{array}{l}(.228) \\
.928 \\
.567 \\
(.666) \\
(.866)\end{array}$ & $\begin{array}{l}.229 \\
.928 \\
.566 \\
.667 \\
.866\end{array}$ & $\begin{array}{l}.228 \\
.926 \\
.566 \\
.666 \\
.867\end{array}$ & $\begin{array}{c}a^{2} \mathrm{P}_{2}-4 \mathrm{R}_{2}^{\circ} \\
a^{3} \mathrm{P}_{2}-2 \mathrm{R}_{2}^{\circ} \\
= \\
-\end{array}$ \\
\hline $\begin{array}{l}\mathbf{3 3 2 3} \\
\mathbf{3 3 1 4} \\
\mathbf{3 2 9 8 \cdot 1 3 3} \\
\mathbf{3 2 8 6} \cdot 755 \\
\mathbf{3 2 8 4} \cdot 688\end{array}$ & $\begin{array}{l}(\cdot 735) \\
(\cdot 742) \\
.134 \\
.757 \\
.589\end{array}$ & $\begin{array}{l}.737 \\
.741 \\
.131 \\
.754 \\
.587\end{array}$ & $\begin{array}{l}.737 \\
.742 \\
\cdot .133 \\
.754 \\
.589\end{array}$ & 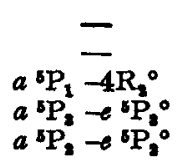 \\
\hline $\begin{array}{l}3280 \\
3271 \cdot 002 \\
3257 \cdot 594 \\
3254 \\
3244 \cdot 190\end{array}$ & $\begin{array}{c}(\cdot 259) \\
.003 \\
.696 \\
(\cdot 362) \\
.190\end{array}$ & $\begin{array}{l}.261 \\
.001 \\
.592 \\
.362 \\
.180\end{array}$ & $\begin{array}{l}.261 \\
.001 \\
.594 \\
.363 \\
\cdot 189\end{array}$ & $\begin{array}{l}a{ }^{5} P_{2}={ }^{b P_{1}}{ }^{\circ} \\
a^{5} P_{3}-4 R_{2}^{\circ} \\
a^{\prime} D_{4}{ }^{\circ}-34 W_{8}\end{array}$ \\
\hline $\begin{array}{l}\mathbf{3 2 3 9} \cdot \mathbf{4 3 6} \\
\mathbf{3 2 3 6 \cdot 2 2 3} \\
\mathbf{3 2 2 5} \cdot \mathbf{7 8 9} \\
\mathbf{3 2 2 2} \cdot 060 \\
\mathbf{3 2 1 7} \cdot \mathbf{3 8 0}\end{array}$ & $\begin{array}{l}.436 \\
.223 \\
.789 \\
.070 \\
.380\end{array}$ & $\begin{array}{l}.437 \\
.222 \\
.790 \\
.069 \\
.381\end{array}$ & $\begin{array}{l}.436 \\
.223 \\
.788 \\
.068 \\
.380\end{array}$ & 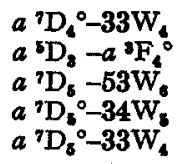 \\
\hline $\begin{array}{l}3215 \cdot 940 \\
3205 \cdot 400 \\
3200 \cdot 475 \\
3196 \cdot 930 \\
3191 \cdot 659\end{array}$ & $\begin{array}{l}.941 \\
.402 \\
.476 \\
.930 \\
.690\end{array}$ & $\begin{array}{l}.940 \\
.400 \\
.474 \\
.930 \\
.660\end{array}$ & $\begin{array}{l}.940 \\
.399 \\
.474 \\
.929 \\
.658\end{array}$ & $\begin{array}{l}a^{7} \mathrm{D}_{2}^{0}-20 \mathrm{~W}_{2} \\
a^{7} \mathrm{D}_{1}^{\circ}-43 \mathrm{~W}_{1} \\
a^{7} \mathrm{D}_{2}^{\circ}-17 \mathrm{~W}^{\circ} \\
a^{7} \mathrm{D}_{4}^{\circ}-25 \mathrm{~W}_{3} \\
a^{6} \mathrm{D}_{4}-a^{3} \mathrm{D}_{2}^{\circ}\end{array}$ \\
\hline $\begin{array}{l}3184 \cdot 896 \\
3178 \cdot 015 \\
3175 \cdot 447 \\
3160 \cdot 658 \\
3157 \cdot 040\end{array}$ & $\begin{array}{l}.896 \\
.014 \\
.447 \\
.660 \\
.040\end{array}$ & $\begin{array}{l}.896 \\
.016 \\
.447 \\
.657 \\
.042\end{array}$ & $\begin{array}{l}.895 \\
.014 \\
.446 \\
.658 \\
.039\end{array}$ & $\begin{array}{l}a^{8} \mathrm{D}_{8}-a^{3} \mathrm{~F}_{8}{ }^{\circ} \\
a^{7} \mathrm{D}_{6}^{\circ}-27 \mathrm{~W}_{4} \\
a^{7} \mathrm{D}_{6}^{\circ}-25 \mathrm{~W}_{8} \\
a^{\prime} \mathrm{D}_{4}^{\circ}-16 \mathrm{~W}_{4} \\
a^{\prime} \mathrm{D}_{4}^{\circ}-13 \mathrm{~W}_{4}\end{array}$ \\
\hline $\begin{array}{l}\mathbf{3 1 4 3} \\
3134 \cdot 111 \\
\mathbf{3 1 1 6} \cdot 633 \\
\mathbf{3 0 9 1} \cdot 578 \\
\mathbf{3 0 8 3 \cdot 7 4 2}\end{array}$ & $\begin{array}{l}-\overline{111} \\
.633 \\
.677 \\
.742\end{array}$ & $\begin{array}{l}-\overline{111} \\
.633 \\
.679 \\
.743\end{array}$ & $\begin{array}{l}.990 \\
.111 \\
.633 \\
.578 \\
.742\end{array}$ & $\begin{array}{l}a^{5} \mathrm{~F}_{3}-c^{5} \mathrm{D}_{4}^{0} \\
a^{5} \mathrm{~F}_{1}-c^{5} \mathrm{D}_{2}^{\circ} \\
a^{3} \mathrm{~F}_{1}-c^{5} \mathrm{D}_{0}^{\circ} 0 \\
a^{5} \mathrm{~F}_{3}-c^{5} \mathrm{D}_{1}^{\circ}\end{array}$ \\
\hline $\begin{array}{l}3075 \cdot 721 \\
3067 \cdot 244 \\
3059 \cdot 086 \\
3057 \cdot 446 \\
3055\end{array}$ & $\begin{array}{l}.721 \\
.246 \\
.086 \\
.446 \\
.263\end{array}$ & $\begin{array}{l}.722 \\
.245 \\
.085 \\
.447 \\
-\end{array}$ & $\begin{array}{l}.720 \\
.243 \\
.087 \\
.445 \\
.263\end{array}$ & 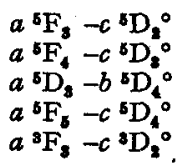 \\
\hline $\begin{array}{l}3047 \cdot 605 \\
3040 \\
3037 \cdot 388 \\
3030 \\
3024\end{array}$ & $\begin{array}{c}\cdot 604 \\
.427 \\
.389 \\
(\cdot 148) \\
.033\end{array}$ & $\begin{array}{l}\frac{.605}{.387} \\
-\end{array}$ & $\begin{array}{l}.606 \\
.428 \\
.389 \\
.149 \\
.033\end{array}$ & $\begin{array}{c}a^{5} \mathrm{D}_{2}-b^{5} \mathrm{D}_{2}^{0} \\
a^{5} \mathrm{~F}_{1}-c{ }^{5} \mathrm{~F}_{5}^{0} \\
a^{5} \mathrm{D}_{1}-b^{3} \mathrm{D}_{2}^{\circ} \\
a^{5} \mathrm{D}_{1}-a^{3} \mathrm{P}_{2}^{0}\end{array}$ \\
\hline \multicolumn{5}{|c|}{83} \\
\hline
\end{tabular}


TABLE 2 (continued)

\begin{tabular}{|c|c|c|c|c|}
\hline$\underset{\lambda}{\text { Recommended }}$ & $\begin{array}{c}\text { Burns- } \\
\text { Walters } \\
\text { calc. }\end{array}$ & Jackson & $\begin{array}{l}\text { Meggers- } \\
\text { Humphreys }\end{array}$ & $\begin{array}{c}\text { Term } \\
\text { combination }\end{array}$ \\
\hline $\begin{array}{l}3015 \\
3009 \\
3003 \\
2999 \cdot 512 \\
2990\end{array}$ & $\begin{array}{c}-572 \\
.534 \\
.512 \\
(\cdot 391)\end{array}$ & $\begin{array}{l}\bar{Z} \\
\overline{.513} \\
-\end{array}$ & $\begin{array}{l}.913 \\
.570 \\
.031 \\
.512 \\
.392\end{array}$ & $\begin{array}{c}a^{5} \mathrm{~F}_{4}-c^{6} \mathrm{~F}_{4}^{0} \\
a^{5} \mathrm{~F}_{3}-c^{6} \mathrm{~F}_{8}^{0} \\
a^{5} \mathrm{~F}_{5}-c^{5} \mathrm{~F}_{5}^{\circ} \\
-\end{array}$ \\
\hline $\begin{array}{l}2987 \cdot 292 \\
2981 \cdot 446 \\
2965 \cdot 255 \\
2959 \\
2957 \cdot 365\end{array}$ & $\begin{array}{l}.293 \\
.446 \\
.255 \\
(.991) \\
.365\end{array}$ & $\begin{array}{l}.291 \\
.446 \\
.255 \\
.365\end{array}$ & $\begin{array}{l}.292 \\
.445 \\
.255 \\
.992 \\
.365\end{array}$ & $\begin{array}{c}a^{6} \mathrm{~F}_{4}-c^{5} \mathrm{~F}_{8}^{\circ} \\
a^{6} \mathrm{D}_{3}-a^{8} \mathrm{P}_{8}^{\circ} \\
a^{5} \mathrm{D}_{0}-b^{5} \mathrm{~F}_{1}^{\circ} \\
a^{5} \mathrm{D}_{1}-b^{6} \mathrm{~F}_{1}^{\circ}\end{array}$ \\
\hline $\begin{array}{l}2953 \cdot 940 \\
2941 \cdot 343 \\
2929 \cdot 008 \\
2920 \\
2912 \cdot 158\end{array}$ & $\begin{array}{l}.940 \\
.343 \\
.008 \\
.691 \\
.158\end{array}$ & $\begin{array}{l}.940 \\
.343 \\
.008 \\
.158\end{array}$ & $\begin{array}{l}.940 \\
.343 \\
.008 \\
.691 \\
.158\end{array}$ & $\begin{array}{l}a^{5} \mathrm{D}_{2}-b^{5} \mathrm{~F}_{2}^{0} \\
a^{5} \mathrm{D}_{2}-b^{5} \mathrm{~F}_{1}^{0} \\
a^{5} \mathrm{D}_{3}-b^{5} \mathrm{~F}_{2} \\
a^{3} \mathrm{~F}_{2}-c^{8} \mathrm{~F}_{2}^{0} \\
a^{5} \mathrm{D}_{4}-b^{5} \mathrm{~F}_{8}^{0}\end{array}$ \\
\hline $\begin{array}{l}2899 \\
2895 \\
2894 \\
2877 \\
2874\end{array}$ & $\begin{array}{c}(\cdot 414) \\
.035 \\
(\cdot 504) \\
.301 \\
.173\end{array}$ & $\begin{array}{l}\bar{z} \\
\overline{-}\end{array}$ & $\begin{array}{l}.416 \\
.035 \\
.505 \\
.300 \\
\cdot 172\end{array}$ & $\begin{array}{l}a^{8} \mathrm{~F}_{3}-c^{3} \mathrm{~F}_{8}^{0} \\
a^{2} \mathrm{~F}_{4}-18 \mathrm{R}_{4}^{0} \\
a^{6} \mathrm{D}_{4}-a^{6} \mathrm{G}_{8}^{\circ}\end{array}$ \\
\hline $\begin{array}{l}2869 \cdot 308 \\
2863 \\
2851 \cdot 798 \\
2845 \\
2838 \cdot 120\end{array}$ & $\begin{array}{l}.308 \\
.864 \\
.799 \\
.595 \\
.122\end{array}$ & $\begin{array}{l}\cdot \frac{.307}{-} \\
\cdot \frac{797}{-120}\end{array}$ & $\begin{array}{l}\cdot 308 \\
\cdot 864 \\
\cdot 797 \\
\cdot 594 \\
\cdot 119\end{array}$ & $\begin{array}{l}a^{5} \mathrm{D}_{3}-a^{5} \mathrm{G}_{4}^{0} \\
a^{5} \mathrm{D}_{2}-a^{5} \mathrm{G}_{3} 0 \\
a^{3} \mathrm{~F}_{1}-b^{5} \mathrm{G}_{2}^{\circ} \\
a^{5} \mathrm{~F}_{3}-c^{5} \mathrm{P}_{2}^{\circ} \\
a^{5} \mathrm{~F}_{2}-b^{5} \mathrm{G}_{2}^{\circ}\end{array}$ \\
\hline $\begin{array}{l}2832 \cdot 436 \\
2823 \cdot 276 \\
2813 \cdot 288 \\
2806 \\
2804 \cdot 521\end{array}$ & $\begin{array}{l}.438 \\
.278 \\
.290 \\
(\cdot 985) \\
.523\end{array}$ & $\begin{array}{l}.436 \\
.276 \\
.288 \\
.985 \\
.521\end{array}$ & $\begin{array}{l}.435 \\
.275 \\
.286 \\
.984 \\
.520\end{array}$ & $\begin{array}{l}a^{5} \mathrm{~F}_{3}-b^{5} \mathrm{G}_{4}^{0} \\
a^{5} \mathrm{~F}_{8}-b^{5} \mathrm{G}_{3}^{\circ} \\
a^{5} \mathrm{~F}_{4}-b^{5} \mathrm{G}_{5}^{\circ} \\
a^{5} \mathrm{~F}_{4}-b^{5} \mathrm{G}_{4}^{\circ}\end{array}$ \\
\hline $\begin{array}{l}2797 \\
2781 \\
2778 \cdot 221 \\
2767 \cdot 523 \\
2763\end{array}$ & $\begin{array}{l}(\cdot 775) \\
\cdot 837 \\
\cdot 224 \\
.524 \\
\cdot 111\end{array}$ & $\begin{array}{l}\overline{-} \\
.220 \\
.525 \\
-\end{array}$ & $\begin{array}{l}.775 \\
.835 \\
\cdot .220 \\
.521 \\
.108\end{array}$ & $\begin{array}{l}a^{5} \mathrm{~F}_{2}-d^{5} \mathrm{D}_{8}^{\circ} \\
a^{5} \mathrm{~F}_{5}-b^{5} \mathrm{G}_{5}^{\circ} \\
a^{5} \mathrm{~F}_{4}-d^{5} \mathrm{D}_{4}^{\circ} \\
a^{5} \mathrm{~F}_{2}-52 \mathrm{R}_{8}^{\circ}\end{array}$ \\
\hline $\begin{array}{l}2755 \\
2749 \\
2746 \\
2746 \\
2739\end{array}$ & $\begin{array}{l}(\cdot 738) \\
(\cdot 322) \\
(\cdot 983) \\
(\cdot 482) \\
(\cdot 546)\end{array}$ & $\begin{array}{l}- \\
- \\
-\end{array}$ & $\begin{array}{l}\cdot 737 \\
.325 \\
.982 \\
.483 \\
.547\end{array}$ & $\begin{array}{l}a{ }^{4} \mathrm{D}_{4}-a{ }^{4} \mathrm{~F}_{8}^{\circ} \\
a^{4} \mathrm{D}_{3}-a \\
a^{4} \mathrm{~F}_{4}^{\circ} \\
a^{4} \mathrm{D}_{3}-a^{4} \mathrm{D}_{8}^{\circ} \\
a^{4} \mathrm{D}_{2}-a^{4} \mathrm{~F}_{8}^{\circ} \\
a^{4} \mathrm{D}_{4}-a^{4} \mathrm{D}_{4}^{\circ}\end{array}$ \\
\hline $\begin{array}{l}2735 \cdot 475 \\
2727 \\
2723 \cdot 577 \\
2718 \\
2714\end{array}$ & $\begin{array}{c}\cdot 477 \\
(\cdot 538) \\
\cdot 577 \\
.436 \\
(\cdot 411)\end{array}$ & $\begin{array}{l}.476 \\
.577 \\
- \\
-\end{array}$ & $\begin{array}{l}.473 \\
.540 \\
.577 \\
.435 \\
.413\end{array}$ & $\begin{array}{l}a^{5} \mathrm{~F}_{4}-d^{5} \mathrm{D}_{8}^{\circ} \\
a^{4} \mathrm{D}_{8}-a{ }^{4} \mathrm{D}_{2}^{\circ} \\
a^{5} \mathrm{D}_{2}-b{ }^{5} \mathrm{P}_{1}^{\circ} \\
a^{5} \mathrm{~F}_{2}-47 \mathrm{R}_{1}^{\circ} \\
a^{6} \mathrm{D}_{4}-a^{6} \mathrm{D}_{8}^{\circ}\end{array}$ \\
\hline $\begin{array}{l}2711 \\
2706 \\
2699 \cdot 107 \\
2689 \cdot 212 \\
2679 \cdot 062\end{array}$ & $\begin{array}{l}.656 \\
.584 \\
.106 \\
.213 \\
.062\end{array}$ & $\begin{array}{l}\overline{ } \\
.108 \\
.212 \\
.063\end{array}$ & $\begin{array}{l}.655 \\
.581 \\
.106 \\
.212 \\
.061\end{array}$ & $\begin{array}{l}a^{5} \mathrm{~F}_{4}-50 \mathrm{R}_{6}^{\circ} \\
a^{5} \mathrm{~F}_{8}-51 \mathrm{R}_{8}^{\circ} \\
a^{5} \mathrm{~s}_{4}-49 \mathrm{R}_{4}^{\circ} \\
a^{5} \mathrm{~b}_{4}-48 \mathrm{R}_{8}^{\circ} \\
a^{5} \mathrm{~s}_{5}-60 \mathrm{R}_{5}^{\circ}\end{array}$ \\
\hline & & & & \\
\hline
\end{tabular}


TABLE 2 (continued)

\begin{tabular}{|c|c|c|c|c|}
\hline$\underset{\lambda}{\operatorname{Recommended}}$ & $\begin{array}{c}\text { Burns- } \\
\text { Walters } \\
\text { calc. }\end{array}$ & Jackson & $\begin{array}{c}\text { Meggers- } \\
\text { Humphreys }\end{array}$ & $\begin{array}{c}\text { Term } \\
\text { combination }\end{array}$ \\
\hline $\begin{array}{l}2673 \\
2662 \\
2651 \\
2647 \\
2644\end{array}$ & $\begin{array}{l}.211 \\
.056 \\
.706 \\
.557 \\
.001\end{array}$ & $\begin{array}{l}\bar{z} \\
\overline{-005}\end{array}$ & $\begin{array}{r}.213 \\
.056 \\
.706 \\
.558 \\
\mathbf{3 . 9 9 7}\end{array}$ & $\begin{array}{l}a^{5} \mathrm{~F}_{1}-c^{3} \mathrm{D}_{1}{ }^{\circ} \\
a^{5} \mathrm{~F}_{3}-c^{3} \mathrm{D}_{2}{ }^{\circ} \\
a^{5} \mathrm{~F}_{3}-b^{3} \mathrm{G}_{4}{ }^{\circ} \\
a^{5} \mathrm{D}_{3}-b^{3} \mathrm{D}_{3}{ }^{\circ} \\
a^{5} \mathrm{~F}_{1}-c^{5} \mathrm{G}_{2}{ }^{\circ}\end{array}$ \\
\hline $\begin{array}{l}2635 \cdot 808 \\
2628 \\
2625 \\
2621 \\
2617\end{array}$ & $\begin{array}{l}.810 \\
(\cdot 292) \\
(\cdot 666) \\
(\cdot 667) \\
(\cdot 615)\end{array}$ & $\begin{array}{l}\frac{.807}{.668} \\
=\end{array}$ & $\begin{array}{l}.808 \\
.292 \\
.666 \\
.669 \\
.616\end{array}$ & $\begin{array}{l}a^{3} \mathrm{~F}_{2}-c^{6} \mathrm{G}_{3}{ }^{\circ} \\
a^{6} \mathrm{D}_{1}-a^{6} \mathrm{D}_{2}^{\circ} \\
a^{6} \mathrm{D}_{4}-a^{6} \mathrm{D}_{3}^{\circ} \\
a^{6} \mathrm{D}_{1}-a^{6} \mathrm{D}_{1}^{\circ} \\
a^{6} \mathrm{D}_{3}-a^{6} \mathrm{D}_{3}^{\circ}\end{array}$ \\
\hline $\begin{array}{l}2613 \\
2611 \\
2598 \\
2585 \\
2584 \cdot 536\end{array}$ & $\begin{array}{l}(.823) \\
(\cdot 873) \\
(.368) \\
(.877) \\
.539\end{array}$ & $\begin{array}{l}-\overline{.872} \\
.8729 \\
.876 \\
.535\end{array}$ & $\begin{array}{l}.824 \\
.872 \\
.369 \\
.875 \\
.535\end{array}$ & $\begin{array}{l}a^{\circ} \mathrm{D}_{2}-a^{8} \mathrm{D}_{1}^{\circ} \\
a^{6} \mathrm{D}_{4}-a^{\circ} \mathrm{D}_{4}^{\circ} \\
a^{\circ} \mathrm{D}_{4}-a^{\circ} \mathrm{D}_{3}^{\circ} \\
a^{\circ} \mathrm{D}_{5}-a^{6} \mathrm{D}^{\circ} \\
a^{\circ} \mathrm{F}_{5}-c^{6} \mathrm{G}_{6}^{\circ}\end{array}$ \\
\hline $\begin{array}{l}2576 \\
2575 \\
2562 \\
2551 \\
2542\end{array}$ & $\begin{array}{l}(\overline{744)} \\
(\cdot 633) \\
(\cdot 000) \\
(\cdot 101)\end{array}$ & $\begin{array}{l}\overline{-} \\
.534 \\
\overline{102}\end{array}$ & $\begin{array}{l}\cdot .103 \\
.744 \\
.535 \\
.094 \\
\cdot 101\end{array}$ & $\begin{array}{c}\overline{-} \\
a{ }^{4} \mathrm{D}_{4}-a \\
- \\
-\end{array}$ \\
\hline $\begin{array}{l}2530 \\
2519 \\
2507 \\
2496 \\
2487\end{array}$ & $\begin{array}{l}(\cdot 691) \\
(\cdot 627) \\
(\cdot 898) \\
(\cdot 534) \\
(\cdot 064)\end{array}$ & $\begin{array}{l}\cdot 692 \\
.900 \\
.533 \\
-\end{array}$ & $\begin{array}{l}.694 \\
.628 \\
.899 \\
.532 \\
.5064\end{array}$ & $\begin{array}{l}\bar{z} \\
\bar{z}\end{array}$ \\
\hline $\begin{array}{l}2474 \\
2468 \\
2465 \\
2457 \\
2453\end{array}$ & $\begin{array}{l}(\cdot 813) \\
(\cdot 878) \\
(\cdot 148) \\
(\cdot 595) \\
(\cdot 472)\end{array}$ & $\begin{array}{l}\cdot 814 \\
.879 \\
\cdot 148 \\
.595 \\
-\end{array}$ & $\begin{array}{l}.813 \\
.878 \\
.148 \\
.596 \\
.475\end{array}$ & $\begin{array}{l}= \\
=\end{array}$ \\
\hline $\begin{array}{l}2447 \cdot 708 \\
2443 \\
2442 \\
2438 \\
2431\end{array}$ & $\begin{array}{l}\cdot 708 \\
\cdot 869 \\
(\cdot \overline{179}) \\
(\cdot 023)\end{array}$ & $\begin{array}{l}\cdot 708 \\
= \\
=\end{array}$ & $\begin{array}{l}.709 \\
.871 \\
.567 \\
.181 \\
.025\end{array}$ & $\begin{array}{c}a^{5} \mathrm{D}_{4}-c^{6} \mathrm{~F}_{8}{ }^{\circ} \\
a^{5} \mathrm{~F}_{5}-25 \mathrm{R}_{4}^{\circ} \\
= \\
=\end{array}$ \\
\hline $\begin{array}{l}2413 \\
2411 \\
2410 \\
2406 \\
2404\end{array}$ & $\begin{array}{l}(\cdot 308) \\
(\cdot 064) \\
(\cdot 518) \\
(\cdot 659) \\
(\cdot 429)\end{array}$ & $\begin{array}{l}.309 \\
.066 \\
.517 \\
.657 \\
-\end{array}$ & $\begin{array}{l}\cdot 309 \\
.066 \\
\cdot 517 \\
\cdot 659 \\
\cdot 430\end{array}$ & $\begin{array}{l}a^{6} \mathrm{D}_{1}-a^{6} \mathrm{~F}_{2}{ }^{\circ} \\
a^{6} \mathrm{D}_{1}-a^{6} \mathrm{~F}_{1}^{\circ} \\
a^{6} \mathrm{D}_{2}-a^{6} \mathrm{~F}_{3}^{\circ} \\
a^{6} \mathrm{D}_{2}-a^{6} \mathrm{~F}_{2}^{\circ} \\
a^{6} \mathrm{D}_{2}-a^{6} \mathrm{~F}_{1}^{\circ}\end{array}$ \\
\hline $\begin{array}{l}2399 \\
2389 \\
2388 \\
2384 \\
2380\end{array}$ & $\begin{array}{l}(\cdot 239) \\
.970 \\
(\cdot 627) \\
(\cdot 385) \\
(\cdot 759)\end{array}$ & $\begin{array}{l}.238 \\
\frac{.625}{.757}\end{array}$ & $\begin{array}{l}.240 \\
.971 \\
.627 \\
.386 \\
.759\end{array}$ & $\begin{array}{l}a^{6} \mathrm{D}_{3}-a^{6} \mathrm{~F}_{3}{ }^{\circ} \\
a^{6} \mathrm{D}_{2}-c^{6} \mathrm{P}_{3}^{\circ} \\
a^{6} \mathrm{D}_{4}-a^{6} \mathrm{~F}_{4}^{\circ} \\
a^{6} \mathrm{~F}_{2}-a^{6} \mathrm{D}_{2}^{0} \\
a^{6} \mathrm{D}_{3}-a^{6} \mathrm{P}_{4}^{\circ}\end{array}$ \\
\hline $\begin{array}{l}2379 \\
2375 \\
2374 \\
2371 \\
2370\end{array}$ & $\begin{array}{l}(\cdot 275) \\
(\cdot 191) \\
.517 \\
.428 \\
(\cdot 495)\end{array}$ & $\begin{array}{l}\cdot 273 \\
= \\
-\end{array}$ & $\begin{array}{l}\cdot 276 \\
\cdot 193 \\
\cdot 517 \\
\cdot 428 \\
\cdot 497\end{array}$ & $\begin{array}{l}a^{4} \mathrm{~F}_{4}-a^{4} \mathrm{D}_{4}^{\circ} \\
a^{4} \mathrm{~F}_{2}-a^{4} \mathrm{D}_{1}^{\circ} \\
a^{5} \mathrm{D}_{0}-c^{5} \mathrm{P}_{1}^{\circ} \\
a^{5} \mathrm{D}_{2}-c^{5} \mathrm{P}_{2}^{\circ} \\
a^{4} \mathrm{~F}_{2}-a^{4} \mathrm{~F}_{2}^{\circ}\end{array}$ \\
\hline \multicolumn{5}{|c|}{83} \\
\hline
\end{tabular}


TABLE 2 (continued)

\begin{tabular}{|c|c|c|c|}
\hline 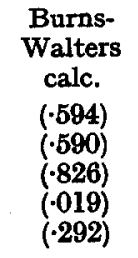 & $\begin{array}{c}\text { Jackson } \\
\overline{-} \\
\overline{825} \\
=\end{array}$ & $\begin{array}{c}\text { Meggers- } \\
\text { Humphreys } \\
.595 \\
.592 \\
.827 \\
.019 \\
.294\end{array}$ & 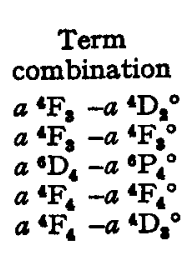 \\
\hline $\begin{array}{l}(.997) \\
(\cdot 104) \\
(\cdot 888) \\
(.279) \\
(.005)\end{array}$ & $\begin{array}{l}\overline{102} \\
\overline{-} \\
.002\end{array}$ & $\begin{array}{l}.997 \\
.104 \\
.889 \\
.280 \\
.005\end{array}$ & $\begin{array}{l}a^{4} \mathrm{~F}_{8}-a{ }^{\circ} \mathrm{F}_{8} \\
a^{\circ} \mathrm{D}_{2}-a^{\circ} \mathrm{P}_{8}^{\circ} \\
a^{\circ} \mathrm{F}_{2}-a \\
a^{\circ} \mathrm{F}_{2} \\
a^{\circ} \mathrm{D}_{1}-a^{\circ} \mathrm{P}_{2}^{\circ} \\
a^{\circ} \mathrm{D}_{1}-a^{\circ} \mathrm{P}_{2}^{\circ}\end{array}$ \\
\hline $\begin{array}{l}(\cdot 796) \\
(\cdot 305) \\
(\cdot 394) \\
.356 \\
\cdot 102\end{array}$ & $\begin{array}{l}.795 \\
.305 \\
.392 \\
- \\
-\end{array}$ & $\begin{array}{l}\cdot 797 \\
.307 \\
.394 \\
.356 \\
.102\end{array}$ & 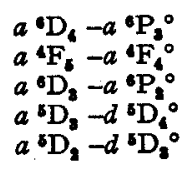 \\
\hline $\begin{array}{l}.996 \\
.578 \\
(.422) \\
.681 \\
.140\end{array}$ & $\begin{array}{l}E \\
=\end{array}$ & $\begin{array}{l}.997 \\
\cdot 579 \\
\cdot 422 \\
\cdot 682 \\
\cdot 140\end{array}$ & $\begin{array}{c}a^{5} \mathrm{D}_{1}-d^{5} \mathrm{D}_{8}^{\circ} \\
a^{5} \mathrm{D}_{1}-45 \mathrm{R}_{2}^{0} \\
a^{\circ}- \\
a^{5} \mathrm{D}_{0}-d^{5} \mathrm{D}_{1}^{\circ} \\
a^{\circ} \mathrm{D}_{1}-52 \mathrm{R}_{8}^{\circ}\end{array}$ \\
\hline $\begin{array}{c}.217 \\
.785 \\
.924 \\
.405 \\
(\cdot 847)\end{array}$ & $\begin{array}{l}\bar{E} \\
\bar{E}\end{array}$ & $\begin{array}{l}.218 \\
.785 \\
.925 \\
.406 \\
.845\end{array}$ & 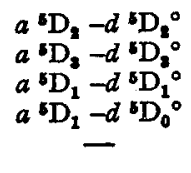 \\
\hline $\begin{array}{l}.522 \\
(\cdot 117) \\
(\cdot 628) \\
.247 \\
.083\end{array}$ & $\bar{E}$ & $\begin{array}{l}.523 \\
.122 \\
.632 \\
.248 \\
.087\end{array}$ & $\begin{array}{c}a^{5} \mathrm{D}_{3}-53 \mathrm{R}_{4}^{\circ} \\
- \\
a^{5} \mathrm{D}_{2}-d^{6} \mathrm{D}_{1}^{\circ} \\
a^{\circ} \mathrm{D}_{2}-d^{5} \mathrm{D}_{2}^{\circ}\end{array}$ \\
\hline $\begin{array}{l}.653 \\
(.094) \\
.024 \\
.087 \\
.066\end{array}$ & $\begin{array}{l}\bar{z} \\
\bar{E}\end{array}$ & $\begin{array}{l}.653 \\
.098 \\
.025 \\
.088 \\
.067\end{array}$ & $\begin{array}{c}a^{5} \mathrm{D}_{1}-51 \mathrm{R}_{2}^{\circ} \\
a^{5} \mathrm{D}_{4}-d^{5} \mathrm{D}_{2}^{\circ} \\
a^{5} \mathrm{D}_{2}-51 \mathrm{R}_{2}^{\circ} \\
a^{5} \mathrm{D}_{2}-49 \mathrm{R}_{1}^{\circ}\end{array}$ \\
\hline $\begin{array}{c}(\cdot 778) \\
.860 \\
.051 \\
(\cdot 390) \\
(\cdot 078)\end{array}$ & $\begin{array}{l}E \\
=\end{array}$ & $\begin{array}{l}.781 \\
.860 \\
.053 \\
.389 \\
.079\end{array}$ & $\begin{array}{c}a^{5} \mathrm{D}_{4}-53 \mathrm{R}_{4}^{\circ} \\
a^{5} \mathrm{D}_{8}-48 \mathrm{R}_{3}^{\circ} \\
a^{\circ} \mathrm{D}_{6}-a \mathrm{~F}^{\circ}\end{array}$ \\
\hline $\begin{array}{l}(\cdot 511) \\
(\cdot 859) \\
(\cdot 122) \\
(\cdot 173) \\
(\cdot 855)\end{array}$ & $\begin{array}{l}z \\
z\end{array}$ & $\begin{array}{l}.511 \\
.861 \\
.125 \\
.177 \\
.858\end{array}$ & 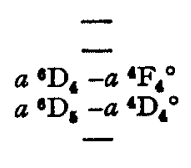 \\
\hline $\begin{array}{l}\cdot 649 \\
\cdot 210 \\
\cdot 168 \\
\cdot 234\end{array}$ & $\begin{array}{l}\bar{E} \\
\bar{E}\end{array}$ & $\begin{array}{l}.651 \\
.627 \\
.211 \\
.170 \\
.234\end{array}$ & 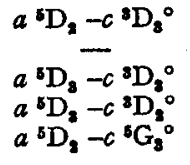 \\
\hline & 84 & & \\
\hline
\end{tabular}


TABLE 2 (continued)

\begin{tabular}{|c|c|c|c|c|}
\hline$\underset{\lambda}{\text { Recommended }}$ & $\begin{array}{c}\text { Burns- } \\
\text { Walters } \\
\text { calc. }\end{array}$ & Jackson & $\begin{array}{l}\text { Meggers- } \\
\text { Humphreys }\end{array}$ & $\begin{array}{c}\text { Term } \\
\text { combination }\end{array}$ \\
\hline $\begin{array}{l}2210 \\
2207 \\
2201 \\
2200 \\
2196\end{array}$ & $\begin{array}{l}.685 \\
.065 \\
.115 \\
.722 \\
.037\end{array}$ & $\begin{array}{l}\bar{z} \\
=\end{array}$ & $\begin{array}{l}.686 \\
.068 \\
.117 \\
.723 \\
.040\end{array}$ & $\begin{array}{l}a{ }^{5} \mathrm{D}_{4}-c^{3} \mathrm{D}_{3}^{\circ} \\
a^{5} \mathrm{D}_{4}-b^{3} \mathrm{G}_{8}^{\circ} \\
a^{5} \mathrm{~b}_{3}-c^{5} \mathrm{G}_{4}^{\circ} \\
a^{5} \mathrm{D}_{1}-d^{5} \mathrm{P}_{8}^{\circ} \\
a^{5} \mathrm{D}_{1}-d^{5} \mathrm{P}_{1}^{\circ} \circ\end{array}$ \\
\hline $\begin{array}{l}2191 \\
2187 \\
2180 \\
2183 \\
2180\end{array}$ & $\begin{array}{l}.200 \\
.190 \\
.888 \\
-.84\end{array}$ & $\begin{array}{l}= \\
\bar{z}\end{array}$ & $\begin{array}{l}.202 \\
.192 \\
.890 \\
.979 \\
.866\end{array}$ & $\begin{array}{l}a{ }^{5} \mathrm{D}_{0}-20 \mathrm{R}_{1}^{\circ} \\
a^{\circ} \mathrm{D}_{2}-d^{5} \mathrm{P}_{1}^{\circ} \\
a^{\circ} \mathrm{D}_{1}-20 \mathrm{R}_{1}^{\circ} \\
a^{\circ}{ }^{\circ} \mathrm{D}_{1}-b{ }^{\circ} \mathrm{P}_{2}^{\circ}\end{array}$ \\
\hline $\begin{array}{l}2176 \\
2173 \\
2172 \\
2165 \\
2164\end{array}$ & $\begin{array}{c}.836 \\
.209 \\
.580 \\
(\cdot 859) \\
.545\end{array}$ & $\begin{array}{l}\bar{z} \\
\bar{z}\end{array}$ & $\begin{array}{l}.837 \\
.212 \\
.581 \\
.861 \\
\cdot 547\end{array}$ & $\begin{array}{l}a^{5} \mathrm{D}_{0}-b^{3} \mathrm{P}_{1}^{\circ} \\
a^{5} \mathrm{D}_{1}-16 \mathrm{R}_{2}^{\circ} \\
a^{\circ} \mathrm{D}_{1}-b^{\circ} \mathrm{P}_{1}^{\circ} \\
a^{\circ} \mathrm{D}_{2}-16 \mathrm{R}_{2}^{\circ}\end{array}$ \\
\hline $\begin{array}{l}2163 \\
2163 \\
2161 \\
2157 \\
2153\end{array}$ & $\begin{array}{c}.858 \\
(\cdot 363) \\
.575 \\
.790 \\
.002\end{array}$ & $\begin{array}{l}z \\
z\end{array}$ & $\begin{array}{l}.860 \\
.368 \\
.577 \\
.792 \\
.004\end{array}$ & $\begin{array}{c}a^{5} \mathrm{D}_{0}-10 \mathrm{R}_{1}^{\circ} \\
- \\
a^{\circ} \mathrm{D}_{1}-d^{3} \mathrm{D}_{2}^{\circ} \\
a^{\circ} \mathrm{D}_{3}-19 \mathrm{R}_{3}^{\circ} \\
a^{\circ} \mathrm{D}_{1}-d^{3} \mathrm{D}_{2}^{\circ}\end{array}$ \\
\hline $\begin{array}{l}2151 \\
2150 \\
2145 \\
2141 \\
2139\end{array}$ & $\begin{array}{l}.096 \\
.180 \\
.186 \\
.714 \\
.684\end{array}$ & $\begin{array}{l}\bar{z} \\
\bar{z}\end{array}$ & $\begin{array}{l}.099 \\
.182 \\
.188 \\
.715 \\
.695\end{array}$ & 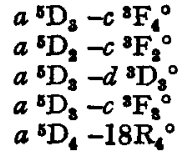 \\
\hline $\begin{array}{l}2138 \\
2135 \\
2132 \\
2130 \\
2115\end{array}$ & $\frac{.688}{. \overline{013}}$ & $\begin{array}{l}\bar{z} \\
\bar{z}\end{array}$ & $\begin{array}{l}.589 \\
.957 \\
.015 \\
.962 \\
.168\end{array}$ & $\begin{array}{l}a^{5} \mathrm{D}_{4}-19 \mathrm{R}_{2}^{\circ} \\
a^{\circ} \mathrm{D}_{4}-c{ }^{3} \mathrm{~F}_{4}^{\circ} \\
a^{\circ} \mathrm{D}_{2}-e^{5} \mathrm{P}_{1}^{\circ}\end{array}$ \\
\hline $\begin{array}{l}2112 \\
2110 \\
2108 \\
2102 \\
2100\end{array}$ & $\begin{array}{l}.965 \\
.955 \\
.350 \\
.794\end{array}$ & $\begin{array}{l}\bar{z} \\
\bar{z}\end{array}$ & $\begin{array}{l}.966 \\
.233 \\
.955 \\
.349 \\
.795\end{array}$ & $\begin{array}{c}a^{5} \mathrm{D}_{0}-e^{5} \mathrm{P}_{1}{ }^{\circ} \\
a^{5} \mathrm{D}_{1}-e^{5} \mathrm{P}_{1} \\
a^{5} \mathrm{D}_{3}-{ }^{5} \mathrm{P}_{3}^{\circ} \\
a^{5} \mathrm{D}_{2}-e^{5} \mathrm{P}_{1}^{\circ}\end{array}$ \\
\hline
\end{tabular}

In Table $\mathrm{I}$, the new measurements are compared with those adopted in 1928 (Trans. I.A.U. 3, 86, 1928) for the overlapping range, 3497 to 3370 A. A similar comparison, made in the I932 report (Trans. I.A.U. 4, 73, I932), suggested slight amendments to a few of the I 928 values but no action was taken. The present comparison, which includes another set of observations, indicates definitely that some of the adopted values should be reduced by o.00I A. In Table 2 the thrice determined values between 3370 and $2447 \mathrm{~A}$. are in remarkably good agreement (except for $2644 \mathrm{~A}$.), but for waves shorter than $2330 \mathrm{~A}$., the Burns and Walters values are systematically $0 \cdot 00 \mathrm{I}_{4} \mathrm{~A}$. lower than those by Meggers and Humphreys. This difference is only $\mathrm{I} / \mathrm{I} 5$ of the phase-dispersion correction which was experimentally determined by Meggers and Humphreys as necessary at 2roo A. for $2 \mathrm{~mm}$. etalons and aluminized interferometer plates. This correction for the spectral change of phase at reflection, which is inherent in the Fabry-Perot interferometer 
method of comparing wave-lengths, may impose a limit on the accuracy with which lines far removed from the primary standard can be measured relative to the latter. For this reason it may be preferable to make such comparisons by a reflectionechelon method (proposed by W. E. Williams, Proc. Phys. Soc. 45, 699, 1933) in which no account need be taken of the variation with wave-length of the phase change at reflection. Regardless of method, a third set of determinations of iron lines in the ultra-violet is required to give the present system of secondary standards a possible extension to $2100 \mathrm{~A}$.

\section{WAVE-LENGTH STANDARDS IN THE EXTREME ULTRA-VIOLET}

No interferometer measurements of wave-lengths shorter than $2100 \mathrm{~A}$. have been reported. For these, and for the entire range of vacuum spectroscopy, it appears advisable to recommend the use of the reflection echelon whose theoretical advantages over the Fabry-Perot interferometer include a constant and possibly higher resolving power, avoidance of differential phase-change corrections, and perfect transparency throughout the extreme ultra-violet.

Other methods of fixing standards in the extreme ultra-violet involve computations based on series formulas or on the combination principle of spectroscopy. Paschen's (Preuss. Akad. I929, 662) calculations of the $\mathrm{H}$ and $\mathrm{He}$ wave-lengths are an illustration of the former, while Shenstone's (Phil. Trans. 235, I95, I936) paper on $\mathrm{Cu}$ II is a fine example of the latter. From terms whose relative values are fixed by interferometer (and grating) measuremients from the infra-red to the ultra-violet at $2100 \mathrm{~A}$., Shenstone was able to calculate the wave-lengths of more than roo $\mathrm{Cu}$ II lines lying between $2000 \cdot 339$ and $685.139 \mathrm{~A}$. These values are thought to have an accuracy varying from $0.003 \mathrm{~A}$. at $1700 \mathrm{~A}$. to $0.0006 \mathrm{~A}$. at $800 \mathrm{~A}$.

In view of the superior quality of secondary standards obtained from the first spectra of the noble gases we might expect to calculate satisfactory standards throughout the extreme ultra-violet if the relative values of the terms for their second and third spectra can be accurately fixed by interferometer observations of the longer waves. An attempt, at the National Bureau of Standards, to carry this through for $\mathrm{A}$ and $\mathrm{Kr}$ failed because none of the conventional light sources emit sufficiently homogeneous spark lines of the noble gases to allow high precision measurement of their wave-lengths.

Since the best-observed constant wave-number differences and cyclical combinations indicate that the combination principle is probably an exact law of atomic spectra, it would seem that the calculation of wave-lengths in this manner would be the ideal method of producing standards in spectral regions unobserved and difficult to observe. However, aside from the experimental difficulty of establishing the relative values of spectral terms with sufficient accuracy, this method may have a limited precision at present on account of its dependence on the dispersion of air, since cyclical combinations leading to extreme ultra-violet standards are based on standard-air wave-lengths which are converted to vacuum values by means of refractive indices. The I932 report (Trans. I.A.U. 4, 59, I932) quoted some new determinations of the index of refraction of air for the primary standard which indicated that the correct value was $I \cdot 0002764$ as compared with $\mathbf{I} \cdot 000275^{8}$ derived from the formula published by Meggers and Peters (B.S. Sci. Pap. 14, 697, I9I8). Although this difference is important for the correct conversion 
of the primary standard from air to vacuum, relative values of wave-lengths will not be affected unless the dispersion data now in common use are shown to be in error.

\section{THE DISPERSION OF AIR}

In Table 3 the calculated refractivity data of Meggers and Peters (loc. cit.) for standard air are compared with values recently indicated by Paschen ( $A n n$. der Phys. (5), 3I, 75, I938), and with values computed from formulas communicated by Sears (vide infra), or by Kösters and Lampe (Phys. Zeit. 35, 223, 1934), or by Pérard (Trav. Bur. int. Poids Mes. 19, 78, 1934).

\section{TABLE 3}

Refractivity of Dry Air at $15^{\circ} \mathrm{C}$. and $760 \mathrm{~mm}$.

\begin{tabular}{|c|c|c|c|c|c|}
\hline \multirow[b]{2}{*}{$\lambda A$} & \multicolumn{3}{|c|}{$(n-1) 10^{7}$} & \multirow[b]{2}{*}{$\begin{array}{l}\text { Kösters } \\
\text { and Lampe }\end{array}$} & \multirow[b]{2}{*}{ Pérard } \\
\hline & $\begin{array}{l}\text { Meggers } \\
\text { and Peters }\end{array}$ & Paschen & Sears & & \\
\hline $\begin{array}{r}2000 \\
3000 \\
4000 \\
5000 \\
6000 \\
7000 \\
8000 \\
9000 \\
10000\end{array}$ & $\begin{array}{l}3255 \cdot 8 \\
2906 \cdot 9 \\
2817 \cdot 1 \\
2781 \cdot 3 \\
2763 \cdot 3 \\
2753 \cdot 0 \\
2746 \cdot 5 \\
2742 \cdot 1 \\
2739 \cdot 1\end{array}$ & $\begin{array}{l}3240 \\
2910 \\
2822 \\
2788 \\
2772 \\
2760 \\
2751 \\
2748 \\
2744\end{array}$ & $\begin{array}{l}3190 \cdot 6 \\
2912 \cdot 6 \\
2826 \cdot 8 \\
2789 \cdot 2 \\
2769 \cdot 2 \\
2757 \cdot 4 \\
2749 \cdot 8 \\
2744 \cdot 6 \\
2740 \cdot 9\end{array}$ & $\begin{array}{l}3216 \cdot 7 \\
2916 \cdot 4 \\
2828 \cdot 0 \\
2790 \cdot 0 \\
2770 \cdot 1 \\
2758 \cdot 4 \\
2750 \cdot 9 \\
2745 \cdot 8 \\
2742 \cdot 2\end{array}$ & $\begin{array}{l}3264 \cdot 7 \\
2920 \cdot 5 \\
2827 \cdot 2 \\
2788 \cdot 8 \\
2769 \cdot 3 \\
2758 \cdot 0 \\
2750 \cdot 7 \\
2745 \cdot 8 \\
2742 \cdot 4\end{array}$ \\
\hline
\end{tabular}

By comparison the first mentioned are too large at $2000 \mathrm{~A}$. and too small for waves longer than $2500 \mathrm{~A}$., the latter difference being greatest near $4000 \mathrm{~A}$. No doubt remains that the dispersion of air should be redetermined, preferably by two or more observers working independently.

Preparations are being made at the National Bureau of Standards to measure refractivities of standard air from 2000 to 12,000 A., taking advantage of twenty years of improvements in light sources, interferometry, and photography. Preliminary observations give a value of 1.0002764 for the primary standard, and confirm the larger positive correction required by blue and green light.

The following report on "Recent Measurements of the Refractive Index of Air at the National Physical Laboratory" was kindly supplied by Mr Sears at the request of Professor Fowler:

"Since I935, work at the National Physical Laboratory has been directed to a determination of definitive values of the refraction and dispersion of dry $\mathrm{CO}_{2}$-free air for the visible spectrum, an exact knowledge of which is essential before a wavelength definition of the units of length can be adopted for practical purposes in metrology. The measurements were made over an optical path equivalent to more than $2 \times 10^{6}$ wave-lengths of red light, at temperatures extending from $12^{\circ} \mathrm{C}$. to $30^{\circ} \mathrm{C}$. and at pressures extending from roo $\mathrm{mm}$. to $800 \mathrm{~mm}$.

"Preliminary calculations have shown that the observed results can be closely represented by the following formulae:

Dry, $\mathrm{CO}_{2}$-free air at $20^{\circ} \mathrm{C}$. and $760 \mathrm{~mm}$., visible spectrum:

$$
\left(n_{20,760}-I\right) I 0^{6}=267 \cdot 872_{5}+\frac{I \cdot 5 I 89}{\lambda^{2} \text { vac. }}+\frac{0 \cdot 0 I 246}{\lambda^{4} \text { vac. }} \text {. }
$$


At temperature $t^{\circ} \mathrm{C}$. and pressure $p m m$. :

$$
\left(n_{t, p}-I\right)=\left(n_{20,780}-\mathrm{I}\right) \frac{p(I+\beta p)}{760(\mathrm{I}+760 \beta)} \cdot \frac{I+20 \alpha}{\mathrm{I}+\alpha t},
$$

where $\beta=0.73 \times 10^{-6}, \alpha=0.003674$ and $\lambda_{\text {vac }}$ is expressed in microns $\left(\mathrm{I} \times 1 \mathrm{IO}^{-6} \mathrm{~m}\right.$.).

"The definitive values given by these equations are in good accordance with those previously obtained by Pérard (Trav. Bur. int. Poids Mes. 19, 66, 1932) at the Bureau International des Poids et Mesures, and by Kösters and Lampe (Phys. Zeit. 35, 223, I934) at the Physikalisch-Technische Reichsanstalt, both of which are very close to the general mean of all determinations since 1857 , and are appreciably higher than those of Meggers and Peters (Bull. Bur. Stand. 14, 697, 1918), which have been generally regarded during the past twenty years as standard values, particularly for spectroscopic purposes.

"Further examination of the complete series of measurements made at the National Physical Laboratory between I935 and i 937 has led to some interesting conclusions, particularly concerning the relation between refractive index and density of air, and some confirmation has been found for D. Berthelot's (Trav. Bur. int. Poids Mes. 13, I907) representations of the characteristic behaviour of gases at low pressures, based on modifications of the equations of state of van der Waals and Clausius. Another interesting feature is that no evidence has been found in support of the relationship suggested by Tilton (J. Research, Nat. Bur. Stand. 13, III, I934) between the refractivity of dry air and sunspot activity, although the experimental work occupied a period when sunspot activity was probably increasing at its maximum rate for the present cycle.

"It may be added that from internal evidence the experimental accuracy of the work is shown to be equivalent to about $\pm I$ part in $10^{8}$ of the value of the refractive index.

"Finally some experiments have been made to determine the effect of moisture upon the refraction and dispersion of dry $\mathrm{CO}_{2}$-free air, as the information at present available upon this subject is rather meagre. From the results obtained, the refraction and dispersion of water vapour at low pressures were calculated, the values found being in reasonably good agreement with those given by C. and M. Cuthbertson (Phil. Trans. Roy. Soc. A, 213, I, I9I4) as the result of an investigation upon steam.

"It is intended to make similar measurements of the refraction and dispersion of dry, $\mathrm{CO}_{2}$-free air for the infra-red and ultra-violet regions; such extensions should provide a more accurate knowledge of the form of the dispersion curve than is at present available for the whole region extending from $2000 \mathrm{~A}$. up to ro,000 A."

\section{NOBLE GAS STANDARDS}

The 8-figure neon and krypton standards adopted in I935 (Trans. I.A.U. 5, 86,87 , I935) continue to serve excellently for spectroscopical and metrological observations of the highest precision, demonstrating that they are reproducible and convenient. C. V. Jackson's final publication of krypton measurements (Phil. Trans. 236, I, 1936) does not affect the adopted standards, but makes available some additional provisional values in the blue and ultra-violet.

The possibility of extending noble gas standards in the ultra-violet has been 
thoroughly investigated by Humphreys (J.Research, Nat. Bur. Stand. 20, I7, I938), who has published 8-figure values for 27 neon lines (3754.2160 to $3369 \cdot 8086 \mathrm{~A}$.), 40 argon lines $(4702 \cdot 3 \mathrm{I} 64$ to $33 \mathrm{I9} \cdot 3446 \mathrm{~A}$ )), and 37 krypton lines (48I2.6367 to 3424.9433 A.). Relative values as tested by the combination principle appear to be correct within I part in $20,000,000$. No other measurements of comparable precision have been made for ultra-violet lines from noble gas spectra, except those of $\mathrm{Kr}$ by C. V. Jackson. These are quoted in Table 4 and compared with Humphreys's values.

\section{TABLE 4}

\section{Wave-Lengths of Ultra-violet Krypton Lines}

\begin{tabular}{|c|c|c|}
\hline \multirow{2}{*}{$\begin{array}{c}\stackrel{\lambda}{\text { Whole number }} \\
\text { A }\end{array}$} & \multicolumn{2}{|c|}{$\lambda$ Fraction A } \\
\hline & $\begin{array}{l}\text { Humphreys } \\
\mathbf{K r} \text { standard }\end{array}$ & $\begin{array}{c}\text { Jackson } \\
\text { Cd standard }\end{array}$ \\
\hline $\begin{array}{l}\mathbf{3 8 4 5} \\
\mathbf{3 8 3 7} \\
\mathbf{3 8 3 7} \\
\mathbf{3 8 1 2} \\
\mathbf{3 8 0 0}\end{array}$ & $\begin{array}{l}\cdot 9778 \\
.8162 \\
.7028 \\
\cdot 2155 \\
.5437\end{array}$ & $\begin{array}{c}\overline{8162} \\
-\overline{0159} \\
.5440\end{array}$ \\
\hline $\begin{array}{l}\mathbf{3 7 9 6} \\
\mathbf{3 7 7 3} \\
\mathbf{3 6 9 8} \\
\mathbf{3 6 7 9} \\
\mathbf{3 6 7 9}\end{array}$ & $\begin{array}{l}.8839 \\
.4241 \\
.0452 \\
.6111 \\
.5609\end{array}$ & $\begin{array}{c}.8840 \\
.4247 \\
.047 \\
-\end{array}$ \\
\hline $\begin{array}{l}3668 \\
3665 \\
3632 \\
3628 \\
3615\end{array}$ & $\begin{array}{l}\cdot 7363 \\
\cdot 3259 \\
\cdot 4896 \\
\cdot 1570 \\
\cdot 4755\end{array}$ & $\begin{array}{c}\cdot 7374 \\
\cdot 3263 \\
-1571 \\
\cdot 4749\end{array}$ \\
\hline $\begin{array}{l}3540 \\
3539 \\
3522 \\
3511 \\
\mathbf{3 5 0 3}\end{array}$ & $\begin{array}{l}.9538 \\
.5416 \\
.6747 \\
.8963 \\
.8981\end{array}$ & $\begin{array}{c}\bar{Z} \\
.675 \\
-\end{array}$ \\
\hline $\begin{array}{l}3502 \\
\mathbf{3 4 9 5} \\
\mathbf{3 4 3 4} \\
\mathbf{3 4 3 1} \\
\mathbf{3 4 2 4}\end{array}$ & $\begin{array}{l}.5537 \\
.9900 \\
.1423 \\
.7217 \\
.9433\end{array}$ & $\begin{array}{c}.9897 \\
= \\
=\end{array}$ \\
\hline
\end{tabular}

Unfortunately the first spectra of the noble gases have, below $3300 \mathrm{~A}$., no strong lines which can be observed conveniently with interferometers. But since the tested ultra-violet lines appear to be of the same high quality as the visible ones it is urged that they be determined as accurately as possible so that they may be substituted for the primary standard when measuring ultra-violet wave-lengths, thus reducing in future measurements the corrections necessary on account of dispersion of phase change, or standard air. 


\section{STANDARD SOLAR WAVE-LENGTHS}

Seven-figure values of wave-lengths $\left(3597^{\cdot 027}\right.$ to $7 \mathbf{1 2 2 \cdot 2 0 6}$ A.) measured in the solar spectrum were adopted as standards in I928 (Trans. I.A.U. 3, 93, I928). Babcock submitted further measurements of red and infra-red lines in I932 (Trans. I.A.U. 4, 83, I932), and in I935 (Trans. I.A.U. 5, 9I, I935). Similar observations have since been made by the Allegheny Observatory and National Bureau of Standards, and a portion of these results are reported in Table 5, together with the Mount Wilson results for the same spectral range. The latter consist of the latest published values $(A p . J .83$, I03, I936) and of additional values supplied by Babcock in time for insertion in the printer's proof of this report. The Mt. Wilson results are based on standards of reference chosen from the " $\mathrm{A}$ " band of terrestrial oxygen as previously determined from neon standards. The unpublished interferometer measurements of Allegheny Observatory and Bureau of Standards embrace many hundreds of lines between 7000 and ro,960 A., but only those between 7569 and $9899 \mathrm{~A}$. are ready for inclusion in this report. All measurements of solar lines by Allegheny Observatory and Bureau of Standards are referred directly to neon standards and standard air. Both sets of data, MW and AO-BS, are for integrated sunlight, and all lines of solar origin are corrected for Doppler-Fizeau displacements. Data for the interval 7569 to $9899 \mathrm{~A}$. are collected in Table 5. Estimates of the probable error are either not available, or omitted to save space, but it may be stated that the AO-BS values represent the means of 5 to 35 determinations. Calculated probable errors are perhaps less informative than a comparison of these independent results. Such a comparison for $229 \mathrm{MW}$ and AO-BS values in Table 5 reveals an average difference of one part in 4,600,000 and an insignificant systematic difference, AO-BS minus MW, of plus one in 29,000,000! In column 2 of Table 5 , seven-figure values are entered for all lines whose independent determinations deviate less than I part in 4,000,000 from the mean. These 229 lines are recommended for adoption as secondary standards.

TABLE 5

Standard Wave-Lengths in the Infra-red Solar Spectrum (7569-9899 A.)

\begin{tabular}{|c|c|c|c|}
\hline Origin & Recommended $\lambda$ & MW & AO-BS \\
\hline $\begin{array}{l}\mathrm{Fe} \\
\mathrm{Ni} \\
\mathrm{Fe} \\
\mathrm{Fe} \\
\mathrm{A}\end{array}$ & $\begin{array}{l}\mathbf{7 5 6 8 \cdot 9 0 6} \\
\mathbf{7 5 7 4} \cdot 048 \\
\mathbf{7 5 8 3} \\
\mathbf{7 5 8 6} \cdot 027 \\
\mathbf{7 5 9 3}\end{array}$ & $\begin{array}{l}.905 \\
.046 \\
.796 \\
.025 \\
-\end{array}$ & $\begin{array}{l}.907 \\
.050 \\
.802 \\
.029 \\
.697\end{array}$ \\
\hline $\begin{array}{l}\text { A } \\
\text { A } \\
\text { A } \\
\text { A } \\
\text { A }\end{array}$ & $\begin{array}{l}7593 \\
7594 \\
7595 \cdot 770 \\
7599 \cdot 462 \\
7601\end{array}$ & $\begin{array}{l}\overline{-} \\
.768 \\
.463 \\
-\end{array}$ & $\begin{array}{l}.996 \\
.512 \\
.772 \\
.462 \\
\cdot .123\end{array}$ \\
\hline $\begin{array}{l}\mathrm{A} \\
\mathrm{A} \\
\mathrm{A} \\
\mathrm{Ni} \\
\mathrm{Ni}\end{array}$ & $\begin{array}{l}7602 \cdot 995 \\
7607 \\
7611 \cdot 194 \\
7616 \cdot 980 \\
7619 \cdot 214\end{array}$ & $\begin{array}{l}.996 \\
\cdot \overline{194} \\
.980 \\
.212\end{array}$ & $\begin{array}{l}.994 \\
.365 \\
.194 \\
.981 \\
.215\end{array}$ \\
\hline
\end{tabular}


TABLE 5 (continued)

\begin{tabular}{|c|c|c|c|}
\hline Origin & Recommended $\lambda$ & MW & AO-BS \\
\hline $\begin{array}{l}\mathbf{A} \\
\mathbf{A} \\
\mathbf{A} \\
\mathbf{A} \\
\mathbf{A}\end{array}$ & $\begin{array}{l}7619 \\
7621 \cdot 802 \\
7625 \cdot 354 \\
7626 \\
\mathbf{7 6 2 9}\end{array}$ & $\begin{array}{l}-\overline{801} \\
\cdot 353 \\
- \\
-\end{array}$ & $\begin{array}{l}\cdot 696 \\
\cdot 803 \\
\cdot 354 \\
\cdot 520 \\
\cdot 092\end{array}$ \\
\hline $\begin{array}{l}\mathbf{A} \\
\mathbf{A} \\
\mathbf{A} \\
\mathbf{A} \\
\mathbf{A}\end{array}$ & $\begin{array}{l}7630 \\
7633 \\
7638 \cdot 306 \\
7642 \\
7647 \cdot 202\end{array}$ & $\frac{\overline{-}}{\frac{\overline{308}}{\cdot \overline{204}}}$ & $\begin{array}{l}\cdot 241 \\
.034 \\
\cdot 305 \\
\cdot 650 \\
\cdot 200\end{array}$ \\
\hline $\begin{array}{l}\mathrm{A} \\
\mathrm{A} \\
\mathrm{Mg} \\
\mathrm{Fe} \\
\mathrm{A}\end{array}$ & $\begin{array}{l}\mathbf{7 6 4 9} \cdot 553 \\
\mathbf{7 6 5 1} \cdot 963 \\
\mathbf{7 6 5 7} \cdot 606 \\
\mathbf{7 6 6 4} \\
\mathbf{7 6 6 5} \cdot 944\end{array}$ & $\begin{array}{l}.552 \\
.963 \\
\cdot 605 \\
\frac{.944}{}\end{array}$ & $\begin{array}{l}.554 \\
.963 \\
.608 \\
.297 \\
.945\end{array}$ \\
\hline $\begin{array}{l}\mathbf{A} \\
\mathbf{A} \\
\mathbf{A} \\
\mathbf{A} \\
\mathbf{S i}\end{array}$ & $\begin{array}{l}7670 \\
7671 \cdot 669 \\
7676 \cdot 565 \\
\mathbf{7 6 7 7 \cdot 6 1 9} \\
\mathbf{7 6 8 0}\end{array}$ & $\begin{array}{l}\cdot 600 \\
.670 \\
\cdot 563 \\
\cdot 618 \\
\cdot 267\end{array}$ & $\begin{array}{l}.605 \\
.668 \\
.567 \\
.620 \\
.272\end{array}$ \\
\hline $\begin{array}{l}\mathbf{A} \\
\mathbf{A} \\
\mathbf{A} \\
\mathbf{A} \\
\mathbf{A}\end{array}$ & $\begin{array}{l}\mathbf{7 6 8 2} \cdot 758 \\
\mathbf{7 6 8 3} \cdot 802 \\
\mathbf{7 6 8 9} \\
\mathbf{7 6 9 0 \cdot 2 1 8} \\
\mathbf{7 6 9 5} \cdot \mathbf{8 3 8}\end{array}$ & $\begin{array}{l}\cdot 756 \\
\cdot 800 \\
\cdot 177 \\
\cdot 217 \\
\cdot 836\end{array}$ & $\begin{array}{l}\cdot 759 \\
.803 \\
\cdot 183 \\
.220 \\
.840\end{array}$ \\
\hline $\begin{array}{l}\text { A } \\
\text { A } \\
\text { A } \\
\text { Fe } \\
\text { Ni }\end{array}$ & $\begin{array}{l}7606 \cdot 869 \\
7702 \\
7703 \\
7710 \\
7714 \cdot 310\end{array}$ & $\begin{array}{l}\cdot 868 \\
\cdot 736 \\
\cdot 754 \\
-\overline{309}\end{array}$ & $\begin{array}{l}.870 \\
.741 \\
.761 \\
.371 \\
.312\end{array}$ \\
\hline $\begin{array}{l}\mathrm{Fe} \\
\mathrm{Ni} \\
\mathrm{Fe} \\
\mathrm{Fe} \\
\mathrm{Fe}\end{array}$ & $\begin{array}{l}7723 \\
7727 \cdot 616 \\
7742 \\
7748 \cdot 284 \\
7751 \cdot 116\end{array}$ & $\begin{array}{l}-\overline{616} \\
.722 \\
.284 \\
\cdot 116\end{array}$ & $\begin{array}{l}.215 \\
.616 \\
.727 \\
.283 \\
.115\end{array}$ \\
\hline $\begin{array}{l}\mathrm{O} \\
\mathrm{Fe} \\
\mathrm{Ni} \\
\mathrm{Ni} \\
\mathrm{Fe}\end{array}$ & $\begin{array}{l}\mathbf{7 7 7 5} \\
\mathbf{7 7 8 0 \cdot 5 6 8} \\
\mathbf{7 7 8 8} \cdot 933 \\
\mathbf{7 7 9 7} \cdot 588 \\
\mathbf{7 8 0 7} \cdot 916\end{array}$ & $\begin{array}{l}-567 \\
.567 \\
.933 \\
.587 \\
.915\end{array}$ & $\begin{array}{l}\cdot 400 \\
.568 \\
.933 \\
.589 \\
.917\end{array}$ \\
\hline $\begin{array}{l}\mathrm{Fe} \\
\mathrm{Al} \\
\odot \\
\mathrm{Fe} \\
\mathrm{A}\end{array}$ & $\begin{array}{l}\mathbf{7 8 3 2} \cdot 208 \\
\mathbf{7 8 3 6} \cdot 130 \\
\mathbf{7 8 4 9} \\
\mathbf{7 8 5 5} \\
\mathbf{7 8 6 4} \cdot \mathbf{4 3 7}\end{array}$ & $\begin{array}{l}.207 \\
\cdot 132 \\
.984 \\
-\overline{.37}\end{array}$ & $\begin{array}{l}.210 \\
.129 \\
.974 \\
.398 \\
.437\end{array}$ \\
\hline $\begin{array}{l}\text { A } \\
\text { A } \\
\text { A } \\
\text { A } \\
\text { A }\end{array}$ & $\begin{array}{l}\mathbf{7 8 6 6} \\
\mathbf{7 8 7 2} \\
\mathbf{7 8 8 0} \\
\mathbf{7 8 8 5} \cdot 014 \\
\mathbf{7 8 8 7} \cdot 117\end{array}$ & $\begin{array}{l}\overline{-} \\
\overline{.014} \\
.117\end{array}$ & $\begin{array}{l}.068 \\
.795 \\
.694 \\
.014 \\
.117\end{array}$ \\
\hline \multicolumn{4}{|c|}{$9 t$} \\
\hline
\end{tabular}


TABLE 5 (continued)

\begin{tabular}{|c|c|c|c|}
\hline Origin & Recommended $\lambda$ & MW & AO-BS \\
\hline $\begin{array}{l}\mathbf{A} \\
\mathbf{A} \\
\mathbf{A} \\
\mathbf{A} \\
\mathrm{Fe}\end{array}$ & $\begin{array}{l}7893 \cdot 512 \\
7896 \\
7901 \\
7908 \\
7912 \cdot 870\end{array}$ & $\frac{.511}{-}$ & $\begin{array}{l}.513 \\
.037 \\
.776 \\
.752 \\
.871\end{array}$ \\
\hline $\begin{array}{l}\mathrm{A} \\
\mathrm{Si} \\
\mathrm{A} \\
\mathrm{A} \\
\mathrm{A}\end{array}$ & $\begin{array}{l}7915 \cdot 634 \\
7918 \\
7920 \cdot 666 \\
7924 \\
7928 \cdot 618\end{array}$ & $\frac{.632}{.664}$ & $\begin{array}{l}.635 \\
.384 \\
.667 \\
.354 \\
.620\end{array}$ \\
\hline $\begin{array}{l}\mathrm{Fe} \\
\mathrm{Fe} \\
\mathrm{Fe} \\
\mathrm{A} \\
\mathrm{A}\end{array}$ & $\begin{array}{l}7937 \cdot 150 \\
7941 \cdot 096 \\
7945 \cdot 858 \\
7953 \\
7958 \cdot 492\end{array}$ & $\begin{array}{l}.149 \\
.096 \\
.857 \\
.491\end{array}$ & $\begin{array}{l}\cdot 151 \\
.096 \\
.859 \\
.820 \\
.492\end{array}$ \\
\hline $\begin{array}{l}\mathrm{A} \\
\mathrm{A} \\
\mathrm{A} \\
\mathrm{A} \\
\mathrm{A}\end{array}$ & $\begin{array}{l}7960 \\
7961 \\
7964 \\
7971 \cdot 522 \\
7975\end{array}$ & $\begin{array}{l}\overline{-} \\
\overline{520} \\
-\end{array}$ & $\begin{array}{l}.739 \\
.621 \\
.353 \\
.524 \\
.003\end{array}$ \\
\hline $\begin{array}{l}\mathrm{A} \\
\mathrm{A} \\
\mathrm{Fe} \\
\mathrm{A} \\
\mathrm{A}\end{array}$ & $\begin{array}{l}7980 \\
7984 \cdot 342 \\
7994 \cdot 488 \\
8000 \cdot 300 \\
8007\end{array}$ & $\begin{array}{c}. \overline{343} \\
.488 \\
.302 \\
-\end{array}$ & $\begin{array}{l}\cdot 465 \\
.341 \\
\cdot 489 \\
.299 \\
\cdot 480\end{array}$ \\
\hline $\begin{array}{l}\mathrm{A} \\
\mathrm{A} \\
\mathrm{Fe} \\
\mathrm{A} \\
\mathrm{A}\end{array}$ & $\begin{array}{l}8012 \cdot 940 \\
8020 \\
8028 \\
8028 \\
8034\end{array}$ & $\begin{array}{l}.940 \\
= \\
=\end{array}$ & $\begin{array}{l}.940 \\
.715 \\
.324 \\
.542 \\
.298\end{array}$ \\
\hline $\begin{array}{l}\mathrm{A} \\
\mathrm{A} \\
\mathrm{A} \\
\mathrm{A} \\
\mathrm{Fe}\end{array}$ & $\begin{array}{l}8036 \cdot 460 \\
8039 \cdot 600 \\
8043 \\
8045 \cdot 530 \\
8046 \cdot 058\end{array}$ & $\begin{array}{l}.460 \\
.598 \\
-531 \\
.056\end{array}$ & $\begin{array}{l}.460 \\
.601 \\
.169 \\
.530 \\
.059\end{array}$ \\
\hline $\begin{array}{l}\mathrm{Fe} \\
\mathrm{A} ? \\
\mathrm{~A} ? \\
\mathrm{~A} \\
\mathrm{Fe}\end{array}$ & $\begin{array}{l}8047 \cdot 625 \\
8059 \\
8063 \cdot 286 \\
8070 \\
8075 \cdot 158\end{array}$ & $\begin{array}{l}\cdot \frac{.625}{.} \\
. \overline{288} \\
\cdot \overline{158}\end{array}$ & $\begin{array}{l}.625 \\
.544 \\
.286 \\
.018 \\
.157\end{array}$ \\
\hline $\begin{array}{l}\mathrm{Fe} \\
\text { A? } \\
\text { A } \\
\text { A? } \\
\text { A }\end{array}$ & $\begin{array}{l}8085 \\
8094 \\
8096 \cdot 580 \\
8103 \cdot 165 \\
8107 \cdot 842\end{array}$ & $\begin{array}{l}-\overline{-} \\
.580 \\
.165 \\
.841\end{array}$ & $\begin{array}{l}\cdot 170 \\
\cdot 274 \\
\cdot 581 \\
\cdot 165 \\
.843\end{array}$ \\
\hline $\begin{array}{l}\text { A } \\
\text { A } \\
\text { A } \\
\text { A } \\
\text { A }\end{array}$ & $\begin{array}{l}8110 \\
8113 \\
8118 \cdot 910 \\
8125 \cdot 445 \\
8130\end{array}$ & $\begin{array}{l}-\overline{-} \\
.908 \\
.444\end{array}$ & $\begin{array}{l}.574 \\
.950 \\
.912 \\
.446 \\
.015\end{array}$ \\
\hline
\end{tabular}


TABLE 5 (continued)

\begin{tabular}{|c|c|c|c|}
\hline Origin & Recommended $\lambda$ & MW & AO-BS \\
\hline $\begin{array}{l}\mathbf{A} \\
\mathbf{A} \\
\mathbf{A} \\
\mathbf{A} \\
\mathbf{A}\end{array}$ & $\begin{array}{l}8130 \\
8133 \cdot 209 \\
8135 \\
8136 \\
8139 \cdot 718\end{array}$ & $\begin{array}{l}\overline{.209} \\
\overline{718}\end{array}$ & $\begin{array}{l}\cdot 463 \\
\cdot 209 \\
.050 \\
\cdot 209 \\
.717\end{array}$ \\
\hline $\begin{array}{l}\mathbf{A} \\
\mathbf{A} \\
\mathbf{A} \\
\mathbf{A} \\
\mathbf{A}\end{array}$ & $\begin{array}{l}8141 \\
8144 \\
8146 \cdot 213 \\
8147 \cdot 188 \\
8149\end{array}$ & $\begin{array}{l}\overline{-} \\
.214 \\
.188 \\
-\end{array}$ & $\begin{array}{l}\cdot 936 \\
\cdot 194 \\
\cdot 212 \\
\cdot 187 \\
\cdot 271\end{array}$ \\
\hline $\begin{array}{l}\mathbf{A} \\
\mathbf{A} \\
\mathbf{A} \\
\mathbf{A} \\
\mathbf{A}\end{array}$ & $\begin{array}{l}8149 \\
8152 \\
8158 \\
8161 \\
8165 \cdot 337\end{array}$ & $\begin{array}{l}\bar{Z} \\
\overline{-338}\end{array}$ & $\begin{array}{l}.692 \\
.499 \\
.024 \\
.434 \\
.336\end{array}$ \\
\hline $\begin{array}{l}\mathbf{A} \\
\mathbf{A} \\
\mathbf{A} \\
\mathbf{A} \\
\mathbf{A}\end{array}$ & $\begin{array}{l}8168 \\
8169 \cdot 386 \\
8174 \\
8177 \cdot 932 \\
8178 \cdot 491\end{array}$ & $\begin{array}{l}\overline{.384} \\
\frac{.932}{.491}\end{array}$ & $\begin{array}{l}\cdot 822 \\
.387 \\
\cdot 683 \\
.932 \\
.491\end{array}$ \\
\hline $\begin{array}{l}\mathbf{A} \\
\mathbf{A} \\
\mathrm{Na} \\
\mathbf{A} \\
\mathbf{A}\end{array}$ & $\begin{array}{l}8181 \cdot 848 \\
8186 \\
8194 \cdot 836 \\
8199 \\
8200 \cdot 694\end{array}$ & $\begin{array}{l}.847 \\
.371 \\
.836 \\
-\overline{.695}\end{array}$ & $\begin{array}{l}.850 \\
.376 \\
.837 \\
.994 \\
.693\end{array}$ \\
\hline $\begin{array}{l}\mathrm{Fe} \\
\mathrm{A} \\
\mathrm{A} \\
\mathrm{A} \\
\mathrm{A}\end{array}$ & $\begin{array}{l}8207 \cdot 749 \\
8212 \cdot 132 \\
8218 \cdot 114 \\
8221 \cdot 553 \\
8225 \cdot 688\end{array}$ & $\begin{array}{l}\cdot 747 \\
\cdot 132 \\
\cdot 112 \\
\cdot 563 \\
\cdot 688\end{array}$ & $\begin{array}{l}.751 \\
.131 \\
.115 \\
.553 \\
.688\end{array}$ \\
\hline $\begin{array}{l}\mathbf{A} \\
\mathbf{A} \\
\mathbf{A} \\
\mathbf{A} \\
\mathrm{Fe}\end{array}$ & $\begin{array}{l}\mathbf{8 2 2 9} \cdot 762 \\
\mathbf{8 2 3 3} \cdot 906 \\
\mathbf{8 2 3 4} \cdot 628 \\
\mathbf{8 2 3 7} \cdot \mathbf{3 4 1} \\
\mathbf{8 2 3 9} \cdot 132\end{array}$ & $\begin{array}{l}.762 \\
.907 \\
.628 \\
.342 \\
\cdot 132\end{array}$ & $\begin{array}{l}.762 \\
.905 \\
.629 \\
.340 \\
\cdot 133\end{array}$ \\
\hline $\begin{array}{l}\text { A } \\
\text { A } \\
\text { Fe } \\
\stackrel{0}{A} \\
\text { A }\end{array}$ & $\begin{array}{l}8239 \cdot 924 \\
8243 \\
8248 \cdot 137 \\
8248 \cdot 802 \\
8252 \cdot 727\end{array}$ & $\begin{array}{l}.925 \\
.139 \\
.800 \\
.727\end{array}$ & $\begin{array}{l}.922 \\
.128 \\
.135 \\
.804 \\
.727\end{array}$ \\
\hline $\begin{array}{l}\mathbf{A} \\
\mathbf{A} \\
\mathbf{A} \\
\mathbf{A} \\
\mathbf{A}\end{array}$ & $\begin{array}{l}8257 \\
8259 \cdot 692 \\
8263 \cdot 445 \\
8269 \\
8272 \cdot 042\end{array}$ & $\begin{array}{l}\overline{.692} \\
.446 \\
\overline{.041}\end{array}$ & $\begin{array}{l}.865 \\
.691 \\
.444 \\
.184 \\
.043\end{array}$ \\
\hline $\begin{array}{l}\text { A } \\
\text { A } \\
\text { A } \\
\text { A } \\
\text { A }\end{array}$ & $\begin{array}{l}\mathbf{8 2 7 3} \\
\mathbf{8 2 7 9} \cdot 600 \\
\mathbf{8 2 8 2} \\
\mathbf{8 2 8 7} \\
\mathbf{8 2 9 4}\end{array}$ & $\begin{array}{l}\overline{600} \\
= \\
=\end{array}$ & $\begin{array}{l}.082 \\
.600 \\
.024 \\
.944 \\
.162\end{array}$ \\
\hline
\end{tabular}


TABLE 5 (continued)

\begin{tabular}{|c|c|c|c|}
\hline Origin & Recommended $\lambda$ & MW & AO-BS \\
\hline $\begin{array}{l}\mathrm{A} \\
\mathrm{A} \\
\mathrm{A} \\
\mathrm{A} \\
\mathrm{A}\end{array}$ & $\begin{array}{l}8295 \\
8300 \cdot 408 \\
8304 \cdot 300 \\
8311 \cdot 956 \\
8313\end{array}$ & $\begin{array}{c}\overline{.407} \\
.301 \\
.955 \\
-\end{array}$ & $\begin{array}{l}.305 \\
.410 \\
.298 \\
.958 \\
.873\end{array}$ \\
\hline $\begin{array}{l}\mathrm{A} \\
\mathrm{A} \\
\mathrm{A} \\
\mathrm{Fe} \\
\mathrm{A}\end{array}$ & $\begin{array}{l}8316 \cdot 224 \\
8318 \\
8321 \\
8327 \cdot 061 \\
8329 \cdot 682\end{array}$ & $\begin{array}{l}.225 \\
\overline{-} \\
.060 \\
.682\end{array}$ & $\begin{array}{l}\cdot 222 \\
\cdot 139 \\
\cdot 244 \\
.062 \\
\cdot 682\end{array}$ \\
\hline $\begin{array}{l}\mathrm{A} \\
\mathrm{Fe} \\
\mathrm{A}(\mathrm{V}) \\
\mathrm{A} \\
\mathrm{A}\end{array}$ & $\begin{array}{l}8333 \cdot 584 \\
8339 \\
\mathbf{8 3 4 2 \cdot 2 9 0} \\
\mathbf{8 3 4 9 \cdot 1 6 2} \\
\mathbf{8 3 5 3}\end{array}$ & $\begin{array}{c}\cdot 584 \\
. \overline{-289} \\
\cdot 160 \\
-\end{array}$ & $\begin{array}{l}\cdot 584 \\
\cdot 420 \\
\cdot 292 \\
\cdot 164 \\
\cdot 653\end{array}$ \\
\hline $\begin{array}{l}\text { A } \\
\text { A } \\
\text { A } \\
\text { Fe } \\
\text { A }\end{array}$ & $\begin{array}{l}8357 \cdot 040 \\
8357 \\
8362 \cdot 302 \\
8365 \\
8367 \cdot 331\end{array}$ & $\begin{array}{l}\frac{.041}{-302} \\
\frac{.332}{.332}\end{array}$ & $\begin{array}{l}.039 \\
.444 \\
.302 \\
.645 \\
.330\end{array}$ \\
\hline $\begin{array}{l}\mathrm{A} \\
\mathrm{A} \\
\mathrm{Fe} \\
\mathrm{A} \\
\mathrm{A}\end{array}$ & $\begin{array}{l}8373 \\
8376 \cdot 381 \\
8387 \\
8394 \cdot 020 \\
8397 \cdot 152\end{array}$ & $\begin{array}{l}\cdot 711 \\
.382 \\
\cdot 782 \\
\cdot 018 \\
\cdot 152\end{array}$ & $\begin{array}{l}\overline{.380} \\
.788 \\
.022 \\
.153\end{array}$ \\
\hline $\begin{array}{l}\mathrm{A} \\
\mathrm{Ti} \\
\mathrm{A} \\
\mathrm{Fe} \\
\mathrm{Ti}\end{array}$ & $\begin{array}{l}8408 \\
8412 \\
8415 \\
8424 \\
8426 \cdot 514\end{array}$ & $\begin{array}{l}\overline{.356} \\
\overline{-} \\
.514\end{array}$ & $\begin{array}{l}\cdot 760 \\
\cdot 367 \\
\cdot 451 \\
\cdot 145 \\
\cdot 514\end{array}$ \\
\hline $\begin{array}{r}\mathrm{Ti} \\
\mathrm{Ti} \\
\mathrm{Fe} \\
-\mathrm{Si} \\
\mathrm{O}\end{array}$ & $\begin{array}{l}8434 \cdot 968 \\
8435 \\
8439 \cdot 581 \\
8443 \\
8446\end{array}$ & $\begin{array}{l}.968 \\
.655 \\
.580 \\
- \\
-\end{array}$ & $\begin{array}{l}.967 \\
-582 \\
.981 \\
.374\end{array}$ \\
\hline $\begin{array}{l}\mathrm{Fe} \\
\mathrm{Fe} \\
\mathrm{Ca}^{+} \\
\mathrm{Si} \\
\mathrm{Fe}\end{array}$ & $\begin{array}{l}8468 \cdot 418 \\
8471 \cdot 744 \\
8498 \\
8502 \\
8514 \cdot 082\end{array}$ & $\begin{array}{c}.418 \\
.744 \\
\frac{-}{.081}\end{array}$ & $\begin{array}{l}.419 \\
.744 \\
.062 \\
.233 \\
.082\end{array}$ \\
\hline $\begin{array}{l}\mathrm{Fe} \\
\mathrm{A} \\
\mathrm{Fe} \\
\mathrm{Si} \\
\mathrm{A}\end{array}$ & $\begin{array}{l}8515 \cdot 122 \\
8519 \\
8526 \cdot 676 \\
8536 \\
8540\end{array}$ & $\begin{array}{l}\frac{.121}{-674} \\
-\end{array}$ & $\begin{array}{l}\cdot 122 \\
.645 \\
.677 \\
\cdot 174 \\
\cdot 812\end{array}$ \\
\hline $\begin{array}{l}\mathrm{Ca}+ \\
\mathrm{Si} \\
\odot \\
\mathrm{Fe} \\
\mathrm{Fe}\end{array}$ & $\begin{array}{l}8542 \\
8556 \cdot 797 \\
8571 \cdot 807 \\
8582 \cdot 271 \\
8592\end{array}$ & $\begin{array}{l}-\overline{795} \\
.809 \\
.271 \\
-\end{array}$ & $\begin{array}{l}.135 \\
.799 \\
.805 \\
.271 \\
.969\end{array}$ \\
\hline
\end{tabular}

94 
TABLE 5 (continued)

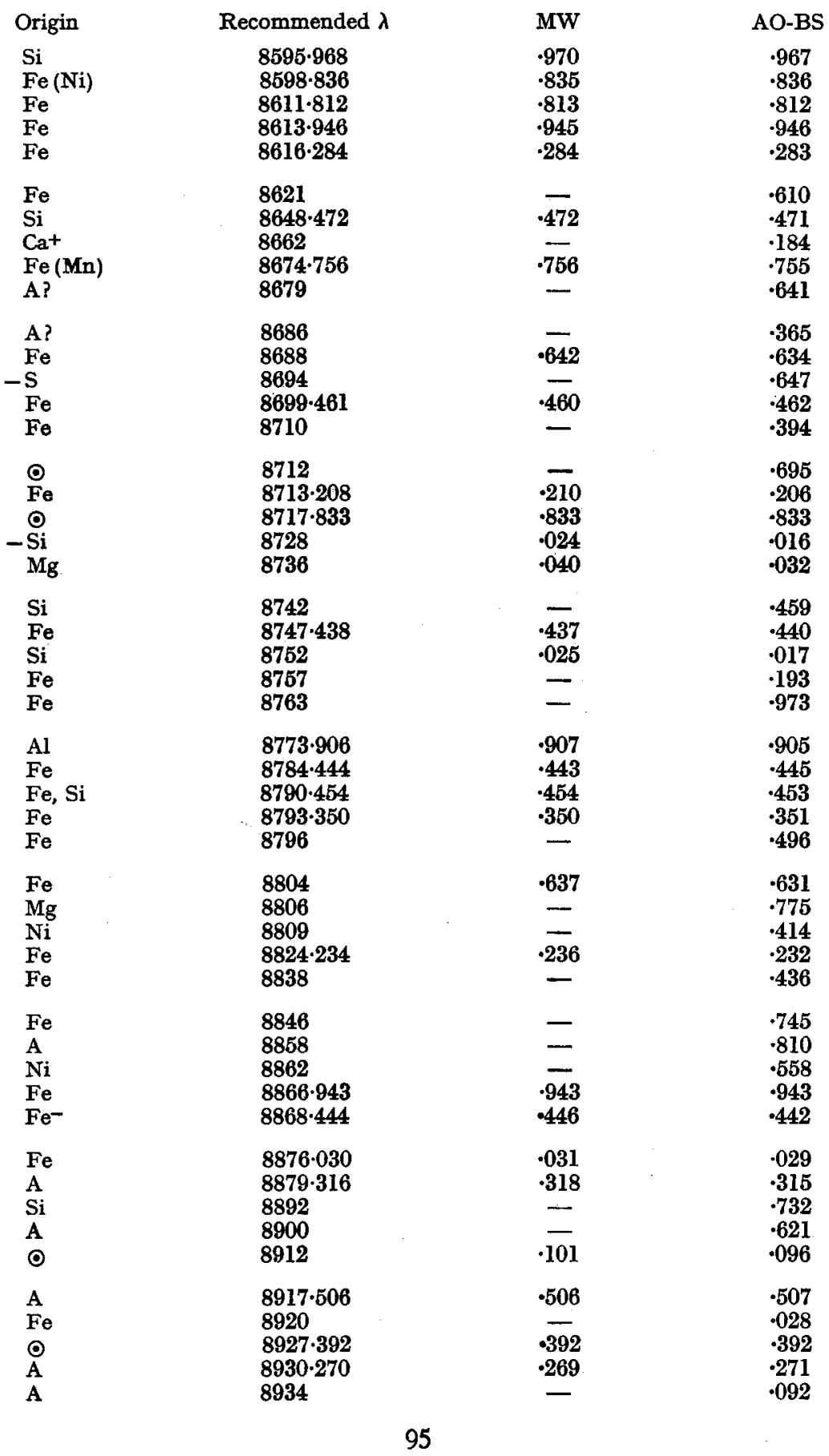


TABLE 5 (continued)

\begin{tabular}{|c|c|c|c|}
\hline Origin & Recommended $\lambda$ & MW & AO-BS \\
\hline $\begin{array}{l}\text { A } \\
\text { A } \\
\text { A } \\
\text { A } \\
\text { A }\end{array}$ & $\begin{array}{l}8940 \\
8942 \\
8946 \cdot 878 \\
8950 \cdot 744 \\
8954\end{array}$ & $\begin{array}{l}\bar{Z} \\
. \overline{878} \\
.746 \\
-\end{array}$ & $\begin{array}{l}\cdot 203 \\
\cdot 343 \\
\cdot 877 \\
\cdot 743 \\
\cdot 305\end{array}$ \\
\hline $\begin{array}{l}\mathbf{A} \\
\mathbf{A} \\
\mathbf{A} \\
\mathbf{A} \\
\mathbf{A}\end{array}$ & $\begin{array}{l}8958 \cdot 402 \\
8963 \cdot 492 \\
8966 \\
8969 \cdot 030 \\
8972\end{array}$ & $\begin{array}{l}.401 \\
.492 \\
. \overline{029} \\
-\end{array}$ & $\begin{array}{l}.402 \\
.492 \\
.413 \\
.032 \\
.897\end{array}$ \\
\hline $\begin{array}{l}\mathbf{A} \\
\mathbf{A} \\
\mathbf{A} \\
\mathbf{A} \\
\mathbf{A}\end{array}$ & $\begin{array}{l}8976 \cdot 424 \\
8981 \\
8986 \\
8993 \cdot 043 \\
9009\end{array}$ & $\begin{array}{l}\frac{.422}{-} \\
.600 \\
.042 \\
-\end{array}$ & $\begin{array}{l}.426 \\
.417 \\
.595 \\
.044 \\
.052\end{array}$ \\
\hline $\begin{array}{l}\mathbf{A} \\
\mathbf{A} \\
\mathbf{A} \\
\mathbf{A} \\
\mathbf{A}\end{array}$ & $\begin{array}{l}9018 \\
9029 \\
9031 \\
9040 \\
9047 \cdot 412\end{array}$ & $\frac{.090}{\frac{.395}{-\overline{411}}}$ & $\begin{array}{l}.097 \\
.396 \\
.406 \\
.093 \\
.414\end{array}$ \\
\hline $\begin{array}{l}\mathbf{A} \\
\mathbf{A} \\
\mathbf{A} \\
\mathbf{A} \\
\mathbf{A}\end{array}$ & $\begin{array}{l}9051 \\
\mathbf{9 0 5 2 \cdot 9 7 4} \\
\mathbf{9 0 6 0 \cdot 4 3 4} \\
9073 \cdot 134 \\
9074 \cdot 306\end{array}$ & $\begin{array}{l}\overline{.972} \\
.435 \\
.132 \\
.308\end{array}$ & $\begin{array}{l}.093 \\
.976 \\
.434 \\
\cdot 136 \\
.305\end{array}$ \\
\hline $\begin{array}{l}\mathbf{A} \\
\mathbf{A} \\
\mathbf{A} \\
\mathbf{A} \\
\mathbf{A}\end{array}$ & $\begin{array}{l}9085 \\
9092 \cdot 482 \\
9101 \\
9105 \cdot 399 \\
9115\end{array}$ & $\begin{array}{c}\cdot 451 \\
\cdot 481 \\
-\overline{401} \\
\cdot 644\end{array}$ & $\begin{array}{l}\cdot 457 \\
\cdot 484 \\
\cdot 512 \\
\cdot 397 \\
\cdot 652\end{array}$ \\
\hline $\begin{array}{l}\text { A } \\
\text { A } \\
\text { A } \\
\text { A } \\
\text { A }\end{array}$ & $\begin{array}{l}9118 \cdot 009 \\
9127 \\
9132 \cdot 443 \\
9140 \cdot 457 \\
9150 \cdot 800\end{array}$ & $\begin{array}{l}.010 \\
.442 \\
.456 \\
.798\end{array}$ & $\begin{array}{l}.008 \\
.820 \\
.444 \\
.458 \\
.801\end{array}$ \\
\hline $\begin{array}{l}\mathbf{A} \\
\mathbf{A} \\
\mathbf{A} \\
\mathbf{A} \\
\mathbf{A}\end{array}$ & $\begin{array}{l}9160 \\
9168 \\
9175 \cdot 249 \\
9178 \cdot 534 \\
9181 \cdot 203\end{array}$ & $\begin{array}{l}\cdot 904 \\
\cdot \overline{249} \\
\cdot 535 \\
\cdot 202\end{array}$ & $\begin{array}{l}-\overline{773} \\
\cdot 249 \\
\cdot 533 \\
\cdot 204\end{array}$ \\
\hline $\begin{array}{l}\mathbf{A} \\
\mathbf{A} \\
\mathbf{A} \\
\mathbf{A} \\
\mathbf{A}\end{array}$ & $\begin{array}{l}9190 \cdot 208 \\
9192 \cdot 568 \\
9199 \\
9205 \cdot 584 \\
9212\end{array}$ & $\begin{array}{l}\cdot 208 \\
.568 \\
-586 \\
-\end{array}$ & $\begin{array}{l}.209 \\
.569 \\
.094 \\
.583 \\
.826\end{array}$ \\
\hline $\begin{array}{l}\mathbf{A} \\
\mathbf{A} \\
\mathbf{A} \\
\mathbf{A} \\
\mathbf{A}\end{array}$ & $\begin{array}{l}9222 \\
9225 \cdot 006 \\
9232 \cdot 750 \\
9234 \\
9238\end{array}$ & $\begin{array}{l}\overline{007} \\
.751 \\
- \\
-\end{array}$ & $\begin{array}{l}.013 \\
.004 \\
.749 \\
.783 \\
.090\end{array}$ \\
\hline
\end{tabular}


TABLE 5 (continued)

\begin{tabular}{|c|c|c|c|}
\hline Origin & Recommended $\lambda$ & $M W$ & AO-BS \\
\hline $\begin{array}{l}\mathbf{A} \\
\hat{A} \\
\mathbf{A} \\
\mathbf{A} \\
\mathbf{A}\end{array}$ & $\begin{array}{l}9240 \\
9251 \cdot 100 \\
9254 \cdot 347 \\
9266 \\
9275 \cdot 072\end{array}$ & $\begin{array}{l}\overline{100} \\
\cdot 347 \\
\overline{.072}\end{array}$ & $\begin{array}{l}.383 \\
.099 \\
.347 \\
.208 \\
.071\end{array}$ \\
\hline $\begin{array}{l}\mathrm{A} \\
\mathrm{A} \\
\mathrm{A} \\
\mathrm{A} \\
\mathrm{A}\end{array}$ & $\begin{array}{l}9289 \cdot 856 \\
9296 \\
9301 \cdot 910 \\
9311 \cdot 734 \\
9314 \cdot 006\end{array}$ & $\begin{array}{l}.855 \\
.910 \\
.735 \\
.007\end{array}$ & $\begin{array}{l}.858 \\
.425 \\
.911 \\
.733 \\
.005\end{array}$ \\
\hline $\begin{array}{l}\mathrm{A} \\
\mathrm{A} \\
\mathrm{Fe} \\
\mathrm{A} \\
\mathrm{A}\end{array}$ & $\begin{array}{l}9320 \cdot 768 \\
9321 \cdot 650 \\
9328 \\
9330 \\
\mathbf{9 3 4 8} \cdot 382\end{array}$ & $\begin{array}{c}.767 \\
.650 \\
-456 \\
.382\end{array}$ & $\begin{array}{l}\cdot 768 \\
.651 \\
\cdot 708 \\
\overline{.381}\end{array}$ \\
\hline $\begin{array}{l}\mathbf{A} \\
\mathbf{A} \\
\mathbf{A} \\
\mathbf{A} \\
\mathbf{A}\end{array}$ & $\begin{array}{l}9360 \\
9361 \cdot 227 \\
9363 \cdot 334 \\
9374 \cdot 280 \\
9400 \cdot 094\end{array}$ & $\begin{array}{l}-\overline{226} \\
.332 \\
.278 \\
.094\end{array}$ & $\begin{array}{l}.595 \\
.228 \\
.335 \\
.281 \\
.095\end{array}$ \\
\hline $\begin{array}{l}\mathbf{A} \\
\mathbf{A} \\
\mathbf{A} \\
\mathbf{A} \\
\mathbf{A}\end{array}$ & $\begin{array}{l}9402 \\
9406 \cdot 904 \\
9412 \\
9420 \\
9420\end{array}$ & $\begin{array}{l}\overline{.903} \\
= \\
-\end{array}$ & $\begin{array}{l}.614 \\
.904 \\
.674 \\
.055 \\
.737\end{array}$ \\
\hline $\begin{array}{l}\text { A } \\
\text { A } \\
\text { A } \\
\text { A } \\
\text { A }\end{array}$ & $\begin{array}{l}9444 \cdot 412 \\
9450 \\
9463 \cdot 992 \\
9467 \\
9472 \cdot 418\end{array}$ & $\begin{array}{l}\frac{.410}{-993} \\
\frac{-}{420}\end{array}$ & $\begin{array}{l}.414 \\
.314 \\
.990 \\
.060 \\
.416\end{array}$ \\
\hline $\begin{array}{l}\text { A } \\
\text { A } \\
\text { A } \\
\text { A } \\
\text { A }\end{array}$ & $\begin{array}{l}9472 \\
9476 \cdot 754 \\
9478 \cdot 884 \\
9483 \cdot 970 \\
9486 \cdot 042\end{array}$ & $\begin{array}{l}\overline{.753} \\
.884 \\
.969 \\
.044\end{array}$ & $\begin{array}{l}.975 \\
.756 \\
.884 \\
.972 \\
.040\end{array}$ \\
\hline $\begin{array}{l}\text { A } \\
\text { A } \\
\text { A } \\
\text { A } \\
\text { A }\end{array}$ & $\begin{array}{l}9491 \\
9503 \\
9504 \cdot 434 \\
9507 \cdot 742 \\
9512 \cdot 630\end{array}$ & $\begin{array}{l}\cdot 526 \\
-435 \\
\cdot 742 \\
\cdot 630\end{array}$ & $\begin{array}{l}.534 \\
.260 \\
.432 \\
.742 \\
.629\end{array}$ \\
\hline $\begin{array}{l}\text { A } \\
\text { A } \\
\text { A } \\
\text { A } \\
\text { A }\end{array}$ & $\begin{array}{l}9514 \\
9526 \\
9533 \cdot 411 \\
9547 \\
9549 \cdot 958\end{array}$ & $\begin{array}{l}\overline{-} \\
\frac{.411}{.957}\end{array}$ & $\begin{array}{l}.484 \\
.872 \\
.411 \\
.096 \\
.958\end{array}$ \\
\hline $\begin{array}{l}\text { A } \\
\text { A } \\
\text { A } \\
\text { A } \\
\text { A }\end{array}$ & $\begin{array}{l}9550 \cdot 962 \\
\mathbf{9 5 5 8} \cdot 836 \\
\mathbf{9 5 7 0} \\
9575 \cdot 680 \\
\mathbf{9 5 8 3}\end{array}$ & $\begin{array}{c}.961 \\
.835 \\
-680 \\
-\end{array}$ & $\begin{array}{l}.963 \\
.836 \\
.325 \\
.680 \\
.592\end{array}$ \\
\hline
\end{tabular}


TABLE 5 (continued)

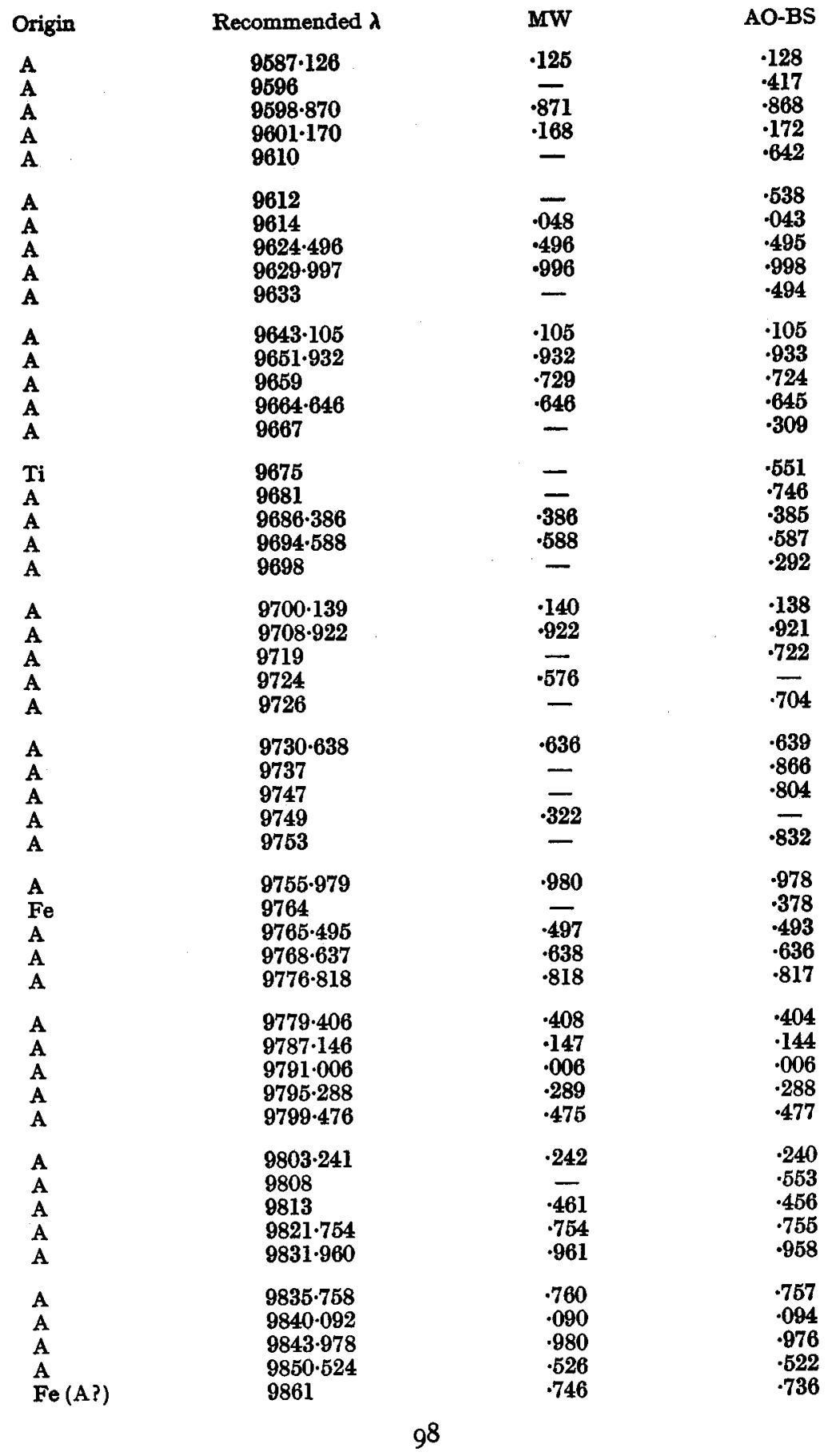


TABLE 5 (continued)

$\begin{array}{lccc}\text { Origin } & \text { Recommended } \lambda & \text { MW } & \text { AO-BS } \\ \mathrm{A} & 9865 & - & \cdot 517 \\ \mathrm{~A} & 9870 & - & .271 \\ \mathrm{~A} & 9873 \cdot 638 & .640 & .636 \\ \mathrm{~A}, \mathrm{Fe} & 9878 \cdot 200 & .200 & .201 \\ \mathrm{Fe} & 9889 \cdot 050 & .052 & .048 \\ \mathrm{Ni}(\mathrm{A} \text { ?) } & 9898 & .972 & .965\end{array}$

Preliminary values of selected lines in the solar spectrum (Io,628 to I2,425 A.) resulting from grating measurements at the Mount Wilson Observatory were presented in the last report (Trans. I.A.U. 5, 92, I935), and a similar but not identical list (I0,628 to I2,I03 A.) was subsequently published $(A p . J .83$, Ir7, I936). Mr Babcock now submits a supplementary list of 30 lines (I2,0I5 to

TABLE 6

Supplementary List of Wave-Lengths and Intensities in the Solar Spectrum (12,015 to $13,376 \mathrm{~A}$.)

$\begin{array}{cccc}\lambda & \text { Intensity } & \lambda & \text { Intensity } \\ 12015 \cdot 21 & 8 & 13000 \cdot 79 & 3 \\ 12047 \cdot 42 & 6 & 13062 \cdot 90 & 10 \\ 12078.06 & 3 & 13077 \cdot 73 & 10 \\ 12088 \cdot 36 & 3 & 13119 \cdot 06 & 5 \\ 12096 \cdot 51 & 3 & 13166 \cdot 64 & 40 \\ 12165 \cdot 26 & 2 & 13184 \cdot 84 & \\ 12223 \cdot 08 & 2 & 13210 \cdot 79 & 3 \\ 12267 \cdot 20 & 3 & 13228 \cdot 60 & 10 \\ 12270 \cdot 65 & 3 & 13237 \cdot 05 & 5 \\ 12604 \cdot 66 & 3 & 13288 \cdot 77 & 5 \\ 12621 \cdot 77 & 3 & 13297 \cdot 88 & 5 \\ 12656 \cdot 93 & 3 & 13321 \cdot 20 & 10 \\ 12723 \cdot 70 & 4 & 13345 \cdot 91 & 5 \\ 12737 \cdot 75 & 1 & 13356 \cdot 56 & 10 \\ 12818 \cdot 10 & 3 & 13376 \cdot 33 & 25 \\ 12970 \cdot 05 & 50^{*} & & 4\end{array}$

13,376 A.), printed here as Table 6 . These preliminary values, at present among the best determined solar wave-lengths exceeding ro,600 A., are given with the hope that they will be measured by other observers co-operating to establish the scale of the infra-red solar spectrum to its present photographic limit.

\section{SPECTROSCOPIC NOTATION}

At its last meeting, this Commission recommended that consideration be given to the standardization of notation for the character and for the quantum description of spectral lines. The suggestion originated with Prof. Kayser who, in compiling spectroscopic data for more than a quarter of a century, encountered a confusion

* Second member of the Paschen series of hydrogen. 
of symbols representing special characteristics or properties of spectral lines in addition to wave-lengths and intensities. A list of such symbols or abbreviations is presented in Table 7 for discussion or adoption.

\section{TABLE 7}

\section{Proposed Notation for the Description of Spectral Lines}

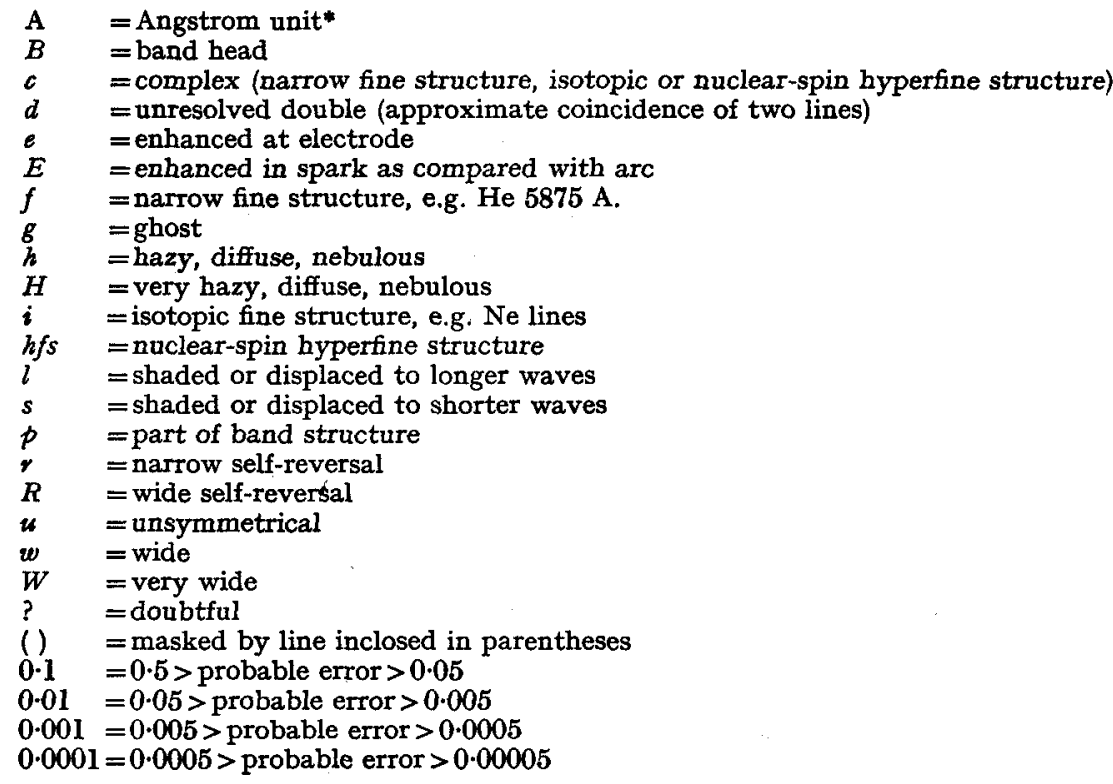

It seems probable that all useful qualitative information about individual spectral lines can be expressed by one or more of these symbols. Thus $B l=$ band head, shaded to longer waves, $c i=$ complex, isotopic fine structure, $g$ ? $=$ ghost, doubtful, $u s=$ unsymmetrical, shaded to shorter waves, etc. The symbol $h f s$ is already in common use for hyperfine structure, and will not be confused with the combination $h, f, s$ because hazy lines cannot reveal narrow fine structure. When the cause of complexity of a spectral line can be recognized, it is informative to distinguish between $f=$ narrow fine structure, $h f s=$ hyperfine structure due to nuclear spin, and $i=$ isotopic fine structure, although more than one cause may be present, e.g. $i, h f s$.

With regard to a satisfactory notation for the representation of spectral terms and for the designation of quantum-interpreted spectral lines, the present state of affairs is most pleasing. Discussion of this subject by a large number of contributors led to the formulation of a system of notation for atomic spectra which was published in 1929 (Phys. Rev. 33, 900, I929). This system completely supplanted earlier notations, employing gothic or greek letters with arbitrary appurtenances, and is now universally used (with minor variations) for the analytical description of spectra. Together with its logical extension to hyperfine structure phenomena (H. E. White, Introduction to Atomic Spectra, 352, McGraw Hill, I934), it has proved to be entirely satisfactory for the complete quantum classification of the data of

* See also page 378 . 
atomic spectra, and should receive the distinction of formal adoption by the International Astronomical Union. The principal variation has been the use of either italic capitals or Roman capitals for spectral term symbols. On account of the advantages of vertical symmetry in placement of super- and sub-scripts and to conform with the rule that italicized symbols do not have quantitative values, the Roman capitals are preferred as symbols for atomic energy levels.

\section{SUMMARY OF RECOMMENDATIONS}

I. It is recommended that the specification for producing the primary standard of wave-length, adopted by the International Committee on Weights and Measures, be formally adopted by the International Astronomical Union.

2. It is recommended that the seven-figure values for ultra-violet iron lines in column I of Tables $I$ and 2 be adopted as secondary standards of wave-length.

3. It is recommended that the seven-figure values for infra-red solar lines presented in column 2 of Table 5 be adopted as solar-spectrum standards of wavelengths.

4. It is recommended that the notation in Table 7 for qualitative description of spectral lines, and the spectral term notation outlined in Phys. Rev. 33, 900, I929, be adopted.

\section{WORK REPORTED IN PROGRESS}

Massachusetts Institute of Technology. The following statement is an abstract of a report submitted by Prof. Harrison:

Grating measurements on about ro,00o iron arc and spark lines between Io,000 and $2000 \mathrm{~A}$. have been made with maximum dispersion of $0.4 \mathrm{~A} . / \mathrm{mm}$., employing the international Pfund arc and Hilger's purest iron. After impurity and ghost lines have been eliminated, many new lines are observed. Testing by means of the combination principle finds the wave-lengths internally consistent to about $0.002 \mathrm{~A}$. on the average.

With the aid of automatic comparators (J.O.S.A. 25, I69, I935; R.S.I. 9, I5, I938) in the past two years more than $8,000,000$ wave-length measurements have been made relative to secondary iron standards and interferometer observations of ultra-violet iron lines by Jackson and by Meggers and Humphreys. The precision appears to be limited by the high intensity and breadth of some of the iron standards when exposed sufficiently to record all of them. This makes it appear desirable to provide at least one set of tertiary standards where the lines are more uniform in intensity and more evenly spaced than those of iron. Consideration of cerium or a similar complex spectrum is suggested.

Allegheny Observatory. Dr Burns reports that interferometer measurements of strontium lines (2282-I0,915 A.) emitted by the vacuum arc at Allegheny Observatory have been completed. Further observations on the solar spectrum are contemplated to supply values in the range $7000-7570 \mathrm{~A}$. and $9900-11,000 \mathrm{~A}$. In the later interval lines of solar origin must be recognized as such in order that the measured wave-lengths may be corrected for relative motion.

National Bureau of Standards. Final preparations are being made for a redetermination of the refractive indices of air for homogeneous wave-lengths distributed throughout the spectrum from 2000 to $12,000 \mathrm{~A}$. Term depressions for the Fe II spectrum are being measured, and the possibility of measuring wave- 
lengths of noble-gas spark lines with precision comparable with that attained for arc lines will be further investigated. Infra-red arc spectra are being photographed, measured and analysed to a limit approaching I3,000 A. Some of these, notably silicon and iron, lead to further identifications of solar lines.

Mount Wilson Observatory. Mr Babcock states that the extensive study of the infra-red solar spectrum has not yet reached a stage warranting its publication, although a large amount of it is practically complete. New impetus has lately been given to this programme through the availability of better instrumental equipment and much more rapid progress is anticipated in the near future.

W. F. MEgGers

President of the Commission

WASHINGTON, D.C.

March I5, I938 